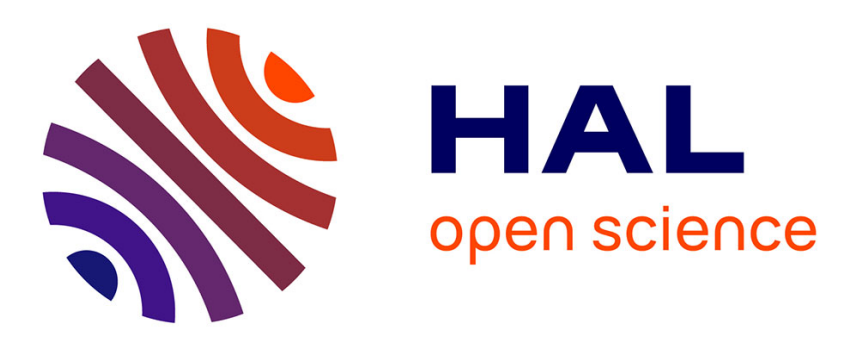

\title{
Motion Control of a Hovering Biomimetic Four-Fin Underwater Robot
}

Taavi Salumäe, Ahmed Chemori, Maarja Kruusmaa

\section{To cite this version:}

Taavi Salumäe, Ahmed Chemori, Maarja Kruusmaa. Motion Control of a Hovering Biomimetic Four-Fin Underwater Robot. IEEE Journal of Oceanic Engineering, 2019, 44 (1), pp.54-71. 10.1109/JOE.2017.2774318 . lirmm-01718243

\section{HAL Id: lirmm-01718243 https://hal-lirmm.ccsd.cnrs.fr/lirmm-01718243}

Submitted on 15 Mar 2018

HAL is a multi-disciplinary open access archive for the deposit and dissemination of scientific research documents, whether they are published or not. The documents may come from teaching and research institutions in France or abroad, or from public or private research centers.
L'archive ouverte pluridisciplinaire HAL, est destinée au dépôt et à la diffusion de documents scientifiques de niveau recherche, publiés ou non, émanant des établissements d'enseignement et de recherche français ou étrangers, des laboratoires publics ou privés. 


\title{
Motion Control of a Hovering Biomimetic 4-fin Underwater Robot
}

\author{
Taavi Salumäe, Ahmed Chemori, and Maarja Kruusmaa
}

\begin{abstract}
U-CAT is a highly maneuverable biomimetic 4-fin underwater robot for operating in confined spaces. Because of its novel mechanical design and specialized purpose, the traditional autonomous underwater robot control methods are not directly applicable on U-CAT. This paper proposes a novel modular control architecture that can be adopted for different application scenarios. Within this framework we implement and test several 2-degree of freedom (DOF) controllers and discuss the test results. Furthermore, we describe and implement an actuation coupling method by prioritizing the selection of DOF with fuzzy membership functions and demonstrate the approach for 3-DOF control. The results show that the proposed DOF prioritization approach helps to improve tracking both in the case of human in the loop and automatic control. Finally, we describe long duration field experiments in realistic environmental conditions.
\end{abstract}

\section{INTRODUCTION}

UTONOMOUS underwater vehicles (AUVs), such as Iver-2 [1], Bluefins [2] and Remus 1 [3], are nowadays widely used in many research areas and industries. For example the oil and gas industry uses them for surveys to locate deposits and to inspect pipelines or rigs [4], navies use them for intelligence and mine countermeasures [5]; and archaeologists use them to locate historical shipwrecks [6]. The large variety of applications of AUVs motivates researchers to develop more and more advanced control systems and technologies for these vehicles. The more advanced control systems in turn shift AUV application towards even more complex underwater environments. In many of these environments, traditional vehicle designs

Taavi Salumäe and Maarja Kruusmaa are with Centre for Biorobotics, Tallinn University of Technology, Tallinn, Estonia, taavi.salumaedtu.ee

Ahmed Chemori is with LIRMM UMR CNRS/Univ. of Montpellier, 161 rue Ada, 34392 Montpellier, France, ahmed. chemorielirmm. fr 


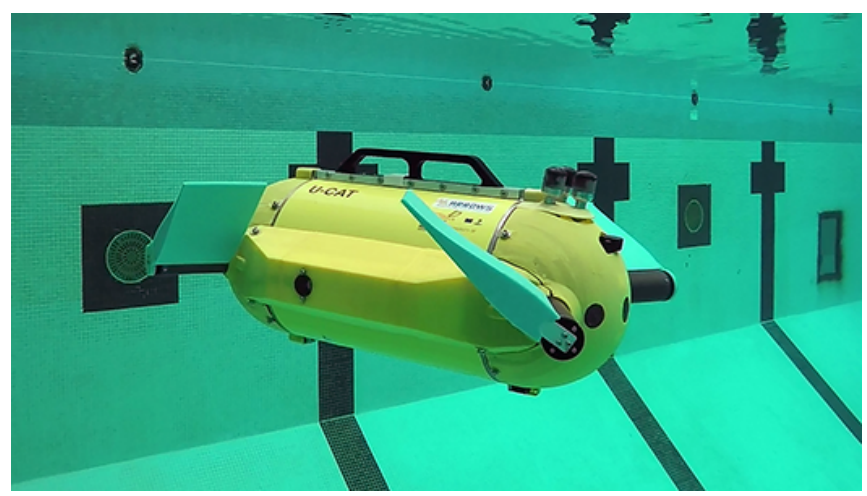

Fig. 1. View of U-CAT biomimetic 4-fin AUV.

are no longer optimal. Traditional AUVs are actuated by propellers and they are relatively large to accommodate high-tech suites. Such vehicles are not well suited to be used in shallow waters, confined areas, in proximity of divers and animals or close to bottom.

The use of biomimetic underwater robots presents an alternative to traditional propoller driven vehicles by using novel thrust creating mechanisms, such as fins, paddels and flippers. Some recent examples of bio-inspired underwater locomotion include mimicking the swimming of fish to create AUVs for mariculture monitoring [7], mimicking the locomotion of snakes in water to develop amphibious vehicles [8] and studying the diving of birds to build aerial-aquatic robots [9]. One class of fin-actuated AUVs consists of multi-fin vehicles often referred to as "turtle-like robots". These vehicles use multiple oscillating fins actuated either in 1 DOF [10]-[13], 2DOFs [14]-[18] or 3DOFs [19]. Most of them use 4 fins, but in some cases the number of actuated fins is different [13]. Some of these studies have a goal to precisely mimic the turtle-like motion [14][16], [19], [20] while in others the biological similarity has lower priority compared to practical feasibility [10]-[13], [18], [21]. With different multi-fin underwater vehicle designs, researchers mainly hope to achieve high maneuverability, quiet and efficient thrust generation as well as reliable or even amphibious locomotion [11], [13], [15]. Alternative locomotion approaches might work better in difficult conditions, such as close to the bottom, next to shore or near water plants and animals.

As the locomotion principle of multi-fin vehicles is very different from that of propelleractuated vehicles, traditional unmanned underwater vehicle (UUV) motion control methods often cannot be directly applied. One main peculiarity of fin-based actuation is the oscillating thrust generation - it is not possible to create constant thrust and this has a strong impact on the control 
stability. The thrust vector of fins is also much less reactive. While the rotation speed of the propeller can usually be changed in a fraction of a second, the thrust of a fin depends on the oscillation amplitude and frequency which cannot be changed equally fast. Moreover, the thrust of fins cannot be as easily and precisely estimated as it is a complex function between oscillation parameters, bending modes and harmonic oscillations of fins and other variables. Next, and likely the biggest difference between highly maneuverable traditional UUVs and multi-fin UUVs, is that the former usually use a separate actuator for controlling each degree of freedom whereas the latter are mostly under-actuated. Multi-fin UUVs instead use different combinations of actuator configurations in order to achieve motion in the available DOFs. This creates strong coupling between the control of different DOFs. For example, when such a vehicle is diving at full speed, the fins are pointed straight up. This configuration completely prevents a surge action to be done at the same time, as this requires the fins to be oriented horizontally.

In this paper we propose a motion control system for biomimetic vehicles actuated by 4 pitching fins. We demonstrate the implementation and functionality of the motion control system on a novel 4-fin biomimetic AUV U-CAT (Fig. 1). U-CAT is an agile 6-DOF maneuverable vehicle developed for autonomous and semi-autonomous inspection of confined spaces, such as shipwrecks, caves, submerged buildings etc. With regards to the mechanical locomotion principle, U-CAT presents a slight departure from previous works; in task/mission directive, the vehicle is fully novel. We propose a control architecture suitable for semi-autonomous and autonomous control of the vehicle, more specifically:

- a modular, easily extensible control architecture that allows rapid and simple implementation and testing of different controllers for different control scenarios.

- comparison of four controllers for simultaneous control of 2 DOFs

- a method for dealing with actuation coupling by prioritizing the action of different DOFs

- simultaneous control of 3 DOFs in 1) manual control with 2 DOF autopiloting, i.e. remotely operated vehicle (ROV) mode; 2) fully autonomous control (AUV mode)

- demonstration and validation of the performance for a real underwater mission

This paper build upon our previous work published on IEEE conferences [22] and [23]. These articles have outlined our approach to the control architecture along with partial test results. This paper extends the conference articles by adding:

- more detailed description of the implementation and validation of the whole control archi- 
tecture,

- model identification of the U-CAT robot,

- description, implementation and experimental comparison of different model-based controllers for selecting the most suitable one for U-CAT,

- experimental validation of the whole system on real underwater robot missions.

The article is structured as follows: section II gives an overview of multi-fin vehicles and their control systems. Section III describes the U-CAT vehicle, its modeling and the model identification. Section IV describes the proposed control architecture including the priority management strategy. Section V compares 4 different controllers to choose the most suitable one for controlling U-CAT. Section VI shows the importance of DOF priority management and validates the control system for remotely operated vehicle mode and fully autonomous mode. Section VII validates the system on two real-life missions. Conclusions are presented in Section VIII.

\section{MUlTi-Fin UNDERWATER VEHICLES AND THEIR CONTROL SYSTEMS}

Most multi-fin vehicles aim to mimic aquatic locomotion of turtles. Marine turtles produce thrust with a complex motion of their forelimbs by using them as rolling and pitching wings creating lift-based forces, as paddles or rows creating mainly drag-based forces, or as a combination of both of them [24]. One of the earliest examples of mimicking such thrust generation can be seen on Turtle 2005 [14] of Kogakuin University that uses rolling and pitching forefins for propulsion and pitching hind-fins for control. The same configuration is used by the robot turtle of Nanyang Technological University [15]. Some other examples are Finnegan the RoboTurtle [16] which uses 4 fins instead of 2 giving the robot a hovering capability, Narotartaruga [19] robot whose fins also include additional yaw motion and Gen series robots of Naval Research Laboratory [20] which use slightly different so called active shape deformation fins [17]. Turtle-like motion has even been mimicked using smart soft composite materials [25]. Some of these robots have better maneuverability than most of the traditional AUVs. However, due to the specifics of the turtle-like actuation, all these robots perform much better in surge motion than in other degrees of freedom. They have none or poor hovering capability and are thus not well suited for tasks such as wall-following or close-up inspection and investigation of confined spaces, in which the vehicle must perform well along each DOF. Another major disadvantage of turtle-mimicking robots is the complexity of multiple-DOF fin actuators. Due to their complex mechanical design such actuators are usually large and less reliable. 
U-CAT generates thrust by using only the pitching motion of fins. This allows us to reduce complexity and size of the fins as each fin is driven only by a single motor. Pitching fins have previously been used also on Madeleine of Nekton Research [10], [26], its commercial successor, an amphibian ROV Transphibian of IRobot [11] and a vehicle developed by Robotics Institute of Beihang University [12]. All these vehicles have 4 pitching fins whose rotation axes are parallel [10] or very close to parallel [11], [12]. Such fin configuration allows to control the robots in maximum 5 DOFs, leaving out the sway motion i.e. they can not move sideways. U-CAT, on the contrary, has a different mechanical configuration that also makes the sideways motion possible, thus giving the vehicle all 6 controllable DOFs.

There are also some other, slightly different vehicles that use pitching fins. An amphibious robot of Peking University [21] uses fins that are actuated by two parallel motors through a special five-bar link mechanism. The mechanism allows the fins to be used for thrust generation or as legs for walking on the ground. Another vehicle of Peking University [18] has fins whose rotation axes can be reoriented using 4 additional motors. This vehicle is extremely highly maneuverable and can be used to control 6 DOFs, but this comes at a price of doubling the number of motors. The amphibious Aqua vehicle of McGill University [13] uses 6 pitching fins instead of 4. The additional two fins help to improve the crawling performance, but are excessive when only aquatic locomotion is desired.

Motion control systems of most of multi-fin vehicles are not as profoundly developed as their mechanical designs, however, there are some exceptions. Many of the authors have only presented open-loop or manual control [12], [14], [15], [18], [21]. Their main contribution lies in the development and analysis of the gait generation, for example using central pattern generators [12], [18], [21]. Some studies have been conducted about the control of non-hover-capable turtlelike vehicles during dominant surge motion. [20] demonstrates model free control for only 1 DOF at the same time, [19] presents model free control of the angular rate for forward swimming stabilization. [16] concentrates mostly on mimicking the fin kinematics and maneuvering of a biological turtle on Finnegan the RoboTurtle, but it presents also results on pitch, roll and yaw control for different dynamically stable and unstable turning maneuvers. To the best of our knowledge, there are no studies that concentrate on the automatic position control of 4-fin hovercapable vehicles. Although [10] claims to have a simultaneous control of different DOFs of the Madeleine vehicle, the authors have not published the control approach or any experimental or simulated results yet. 
Advanced position control has been developed for a 6-fin amphibious Aqua vehicle [13]. It is somewhat similar to the U-CAT in terms of using only pitching fins and being hover-capable. [27] and [28] present modeling and model-based control of Aqua, but only for a single simultaneous DOF. In [29] authors also demonstrate multi-DOF control using a combination of model-free PD and PI controllers for yaw, roll, pitch and heave. They avoid coupling problems by using gain scheduling depending on the surge speed. Even though authors have reported good trajectory following, this was achieved by tuning an array of 45 control parameters, making the developed control approach complex and inextensible. Authors have tried to simplify the process by using online gain adaption algorithms [30], however the presented results show large deviations from desired attitude.

As it can be seen from the background overview, many different fin-actuated vehicles have been developed in recent years. However, the control of these vehicles, especially the position control of hover-capable vehicles is relatively little studied.

\section{U-CAT VEHICLE}

U-CAT is a biomimetic underwater vehicle designed for autonomous and semi-autonomous inspection of confined areas, such as shipwrecks, caves and man-made underwater structures. It was developed in the framework of the European Commission funded research project ARROWS [31]. The aim of ARROWS project was to adapt and develop low-cost AUV technologies to significantly reduce the cost of archaeological operations. U-CAT was designed specifically to meet archaeologists' requirements for unmanned shipwreck penetration. It uses fin-based locomotion to achieve high maneuverability and for quiet and safe locomotion. One unique feature of U-CAT compared to other mentioned 4-fin vehicles lies in its fin configuration. The fins are placed such that a thrust force can be created in all the 6 DOFs. This includes sway, which cannot be controlled with other vehicles using 1 DOF pitching fins, but which is extremely useful for various video inspection tasks such as wall following. More detailed design justification and the description of vehicle are presented in [32]. Some preliminary control results for 1 degree of freedom using non-model-based controllers are published in [22].

U-CAT is $56 \mathrm{~cm}$ long and weights approximately $19 \mathrm{~kg}$. Its four fins are actuated by $60 \mathrm{~W}$ Maxon brushless DC motors driven by Maxon Epos motor drivers using sensorless back-EMF feedback control. The vehicle has an internal battery allowing at least 6 hours of autonomous operation. The whole control architecture is developed on an internal single-board computer 


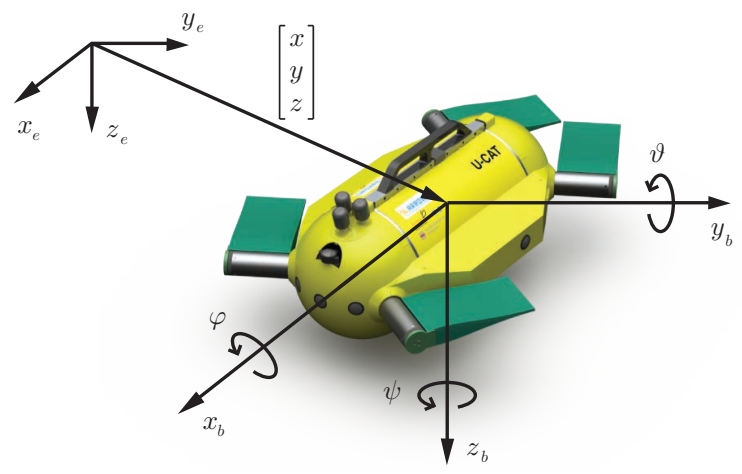

Fig. 2. Coordinate systems used in this study. $\left\{x_{b}, y_{b}, z_{b}\right\}$ is the body-fixed coordinate frame and $\left\{x_{e}, y_{e}, e_{b}\right\}$ is the earth-fixed coordinate frame. Robot's position is presented as a vector of positions in earth-fixed frame $\boldsymbol{\eta}=[x, y, z, \varphi, \vartheta, \psi]^{T}$

with ARM Cortex A9 quad-core $1 \mathrm{Ghz}$ processor. We use a modular development approach with ROS middleware. The on-board computer communicates with a remote PC through a custom connectorless tether mounted on top of the vehicle. In the end of the tether there is a waterproofed $\mathrm{WiFi}$ router, creating an ultra short range underwater WiFi link. We use the remote PC only for forwarding remote controller actions in ROV mode, and accessing the on-board computer for debugging. As a remote controller we used a Sony Playstation DualShock 3.

In this paper we use the following sensors of U-CAT:

- Invensense MPU-6050 IMU for measuring the robot's attitude. In the current study only yaw measurement is directly used as a feedback in control loop. The roll and pitch measurements are used only for coordinate transformations. The sampling frequency is $5 \mathrm{~Hz}$.

- GEMS 3101 analog output pressure sensor with 18-bit DAQ for measuring depth. The sampling frequency is $10 \mathrm{~Hz}$ and the resolution is $0.6 \mathrm{~mm}$.

U-CAT has some additional devices not used in this study, but which will be used in further developments: Applicon acoustic modem for underwater communication and localization; two active buoyancy control modules; custom-developed hydrophone array for acoustic beacon localization; custom-made echosounder array for close-distance obstacle avoidance; Point Grey Chameleon camera; and various system health monitoring sensors.

\section{A. Kinematics}

U-CAT has four fins placed at 30 degree angle with respect to the vehicles center-line on the horizontal plane. Each fin $i$ is separately actuated using a sinusoidal input with parameters: 


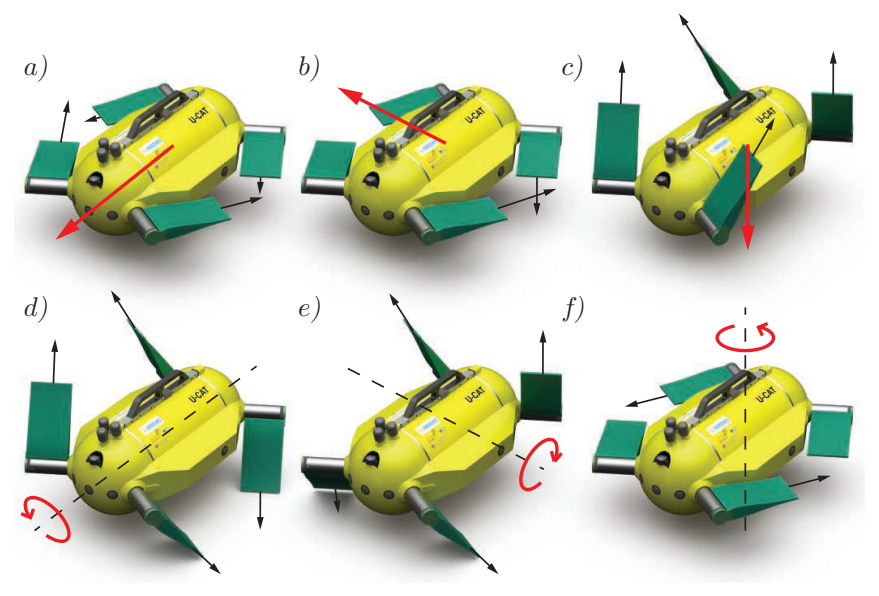

Fig. 3. Motions of the U-CAT in the hovering mode: a) surge; b) sway; c) heave; d) roll; e) pitch; $f$ ) yaw.

$A_{i}$ - oscillation amplitude; $f_{i}$ - oscillation frequency and $\Phi_{i}$ - oscillation offset. The oscillation amplitude and frequency modify the magnitude of thrust while the oscillation offset modifies the direction of thrust. By combining the motions of all four fins it is possible to move the robot in all the three directions and to rotate the robot around all the three axes (Fig. 3).

We have identified 2 main modes of locomotion which we call 1) cruise mode and 2) hovering mode. In cruise mode the fins are generally pointing in the same direction (as illustrated on Fig. 2 for surge motion). The surge motion in cruise mode is achieved by actuating all the fins at the same time. The robot can be oriented by slightly modifying the offset or thrust of some of the fins. The cruise mode is useful when fast forward motion is desired, however, it is not practical for slow maneuvering and hovering at single spot. The reason is that it is not possible to create thrust forces in any direction without reorienting fins. For example when the vehicle is swimming forward and then needs to slightly move backwards, it would have to reorient all its 4 fins 180 degrees, making it impossible to precisely keep station. To avoid this problem during tasks that require precise maneuvering, such as close-up inspection of an artifact, we use the hovering mode. During hovering mode the front and back fins are generally pointing towards each other. Such a fin configuration allows for force in any direction and torque around any axis to be created without fin reorientation (see Fig. 3).

The current article concentrates only on the control of the robot in the hovering mode. The oscillation frequency of the fins is fixed at an experimentally identified optimum value and the desired thrust is being achieved by actively changing the actuation amplitude. The fin oscillations 
are synchronized to have a zero phase shift. Precise synchronization is important to reduce irregular motions of the vehicle resulting from oscillating thrust vectors. When the fins are all oscillating in the same phase, the vehicle oscillates mostly in the heave direction.

\section{B. Dynamics}

We model the rigid body dynamics of the vehicle in 6 DOFs using Fossen's robot-like vectorial model of marine craft [33]. The Fossen's model is well suited for computer implementation and control systems design. Therefore it is widely used in underwater robotics, but also in submarine and surface vessel studies. The 6 DOF model is represented as:

$$
\begin{gathered}
M \dot{\nu}+C(\nu) \nu+D(\nu) \nu+g(\eta)=\tau \\
\dot{\eta}=J(\eta) \nu
\end{gathered}
$$

where $\boldsymbol{\eta}=[x, y, z, \varphi, \vartheta, \psi]^{T}$ is the vector of positions and orientations in the earth-fixed frame, $\boldsymbol{\nu}=[u, v, w, p, q, r]^{T}$ is the vector of linear and angular velocities in the body-fixed frame and $\boldsymbol{J}(\boldsymbol{\eta}) \in \mathbb{R}^{6 \times 6}$ is the transformation matrix mapping from the body-fixed frame to the earthfixed frame. The systems coordinates are presented on Fig. 2. $M=M_{R B}+M_{A}$ is the system inertia matrix, where $M_{R B}$ is the inertia matrix of the vehicle and $M_{A}$ describes the added mass resulting from the accelerating volume of water surrounding the robot. $C(\nu)=C_{R B}+C_{A}$ is the Coriolis-centripetal matrix which again takes into account the rigid body and the added mass. $\boldsymbol{D}(\boldsymbol{\nu})$ is the damping matrix, $\boldsymbol{g}(\boldsymbol{\eta})$ is the vector of gravitational/buoyancy forces and moments and $\boldsymbol{\tau}=[X, Y, Z, K, M, N]^{T}$ is the vector of control inputs. $\boldsymbol{\tau}$ can be represented as a resultant force composed of forces $\boldsymbol{F}_{\boldsymbol{i}}$ with which each fin $i$ acts on the rigid body.

\section{Fin forces}

To successfully control the vehicle using model-based approaches, we need to know the relationship between the fin actuation parameters and the generated thrust force. The forces generated by the fins depend on complex interactions between the viscoelastic body and the hydrodynamics of the surrounding water. We have previously studied the analytical modeling of these interactions [34], however, due to complex nature of the system, analytical models are either too imprecise or not suitable for using in real-time control. Therefore, we create an empirical model of the fin thrust. 


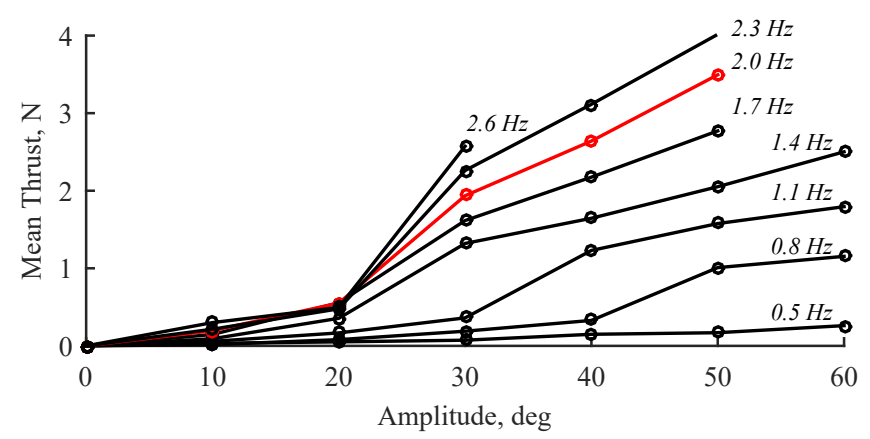

Fig. 4. Mean fin thrust at different actuation parameters. Red marks the frequency of $2 \mathrm{~Hz}$ which we used in our control experiments.

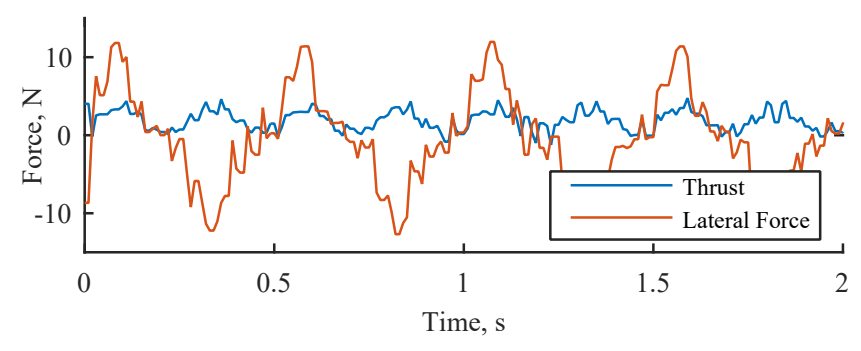

Fig. 5. Thrust and lateral forces generated by the fin while actuated at $2.0 \mathrm{~Hz}$ with the amplitude of $30 \mathrm{deg}$

The average magnitude $F_{i}$ of thrust $\boldsymbol{F}_{\boldsymbol{i}}$ of each fin $i$ is described as

$$
F_{i}=\xi\left(A_{i}, f_{i}\right)
$$

where $A_{i}$ is the oscillation amplitude and $f_{i}$ is the oscillation frequency. We identified the relationship experimentally by mounting one of the U-CAT's fin modules on a waterproof 4-axis force/torque sensor and actuating it with different amplitudes and frequencies. Fig. 4 plots the average thrust during $10 \mathrm{~s}$ experiment period. We fitted the experimental results to gain the relation (3).

To illustrate the strong oscillating nature of the fin locomotion, Fig. 5 plots the example of forces generated by the fins over 2 oscillation periods. The plot shows that the fins do not generate fluctuating forces only in the desired direction. They also generate lateral forces whose amplitude is occasionally exceeding the amplitude of thrust. Large lateral forces are the main reason why the fin motion has to be precisely synchronized. The oscillating motion also has to be taken into consideration during controller implementation as it can initiate strong instability. 


\section{Model identification}

The initial dynamic parameters in (1) were found using both analytical and computational methods. Using experimental model identification, the most important of these parameters were adjusted.

The geometrical properties of the vehicle are found from a detailed 3D model drawn in SolidWorks CAD software. The model includes all the internal and external components of the robot and also the density of the larger components. The 3D model allowed us to precisely calculate all the components of the U-CAT's inertia matrix $M_{R B}$, the Coriolis-centripetal matrix $C_{R B}$, and the gravity and buoyancy vector $\boldsymbol{g}(\boldsymbol{\eta})$. The damping is modeled as a hydrodynamic drag. The drag coefficients in the damping matrix $D(\nu)$ are found using a computational fluid dynamics package called Solidworks Flow Simulation. The inertia $M_{A}$ and Coriolis-centripetal matrix $\boldsymbol{C}_{\boldsymbol{A}}$ of the added mass are calculated with the help of strip theory [35] using simplified geometrical representation of the vehicle. Strip theory is a widely used approach in marine engineering. It can be used to find an added mass of closed 2D contours. The added mass of contours is summed to find the added mass of the whole body.

To achieve higher modeling precision along the most important DOFs, we experimentally identified surge, sway, heave and yaw related components of the added mass inertia matrix $\boldsymbol{M}_{\boldsymbol{A}}$ and drag matrix $\boldsymbol{D}(\boldsymbol{\nu})$. We actuated the robot with a constant known force in a single DOF at the same time and measured the response. To measure the depth response we used the pressure sensor, and for yaw, surge and sway response we used the experimental setup described in Fig. 6. The robot was mounted on a highly buoyant bar to constrain its depth, roll and pitch motions. On the bar there were two contrast markers which were automatically tracked from the video recorded by the calibrated over-head camera. The custom-made tracking software implemented in LabVIEW calculated the vehicle's coordinates on the $x-y$ plane. The robot itself was precisely tuned to be neutrally buoyant to minimize the effect of the bar on the system dynamics. The bar was only slightly touching the water surface.

As during each experiment the robot is moving only along a single axis, the dynamics can be written in a single DOF. For example for the x-axis

$$
\left(M_{x}^{R B}+M_{x}^{A}\right) \dot{u}+D_{x} u=\tau_{x}
$$

The velocity $u$ and acceleration $\dot{u}$ are taken from the experimental results, total fin thrust $\tau_{x}$ 


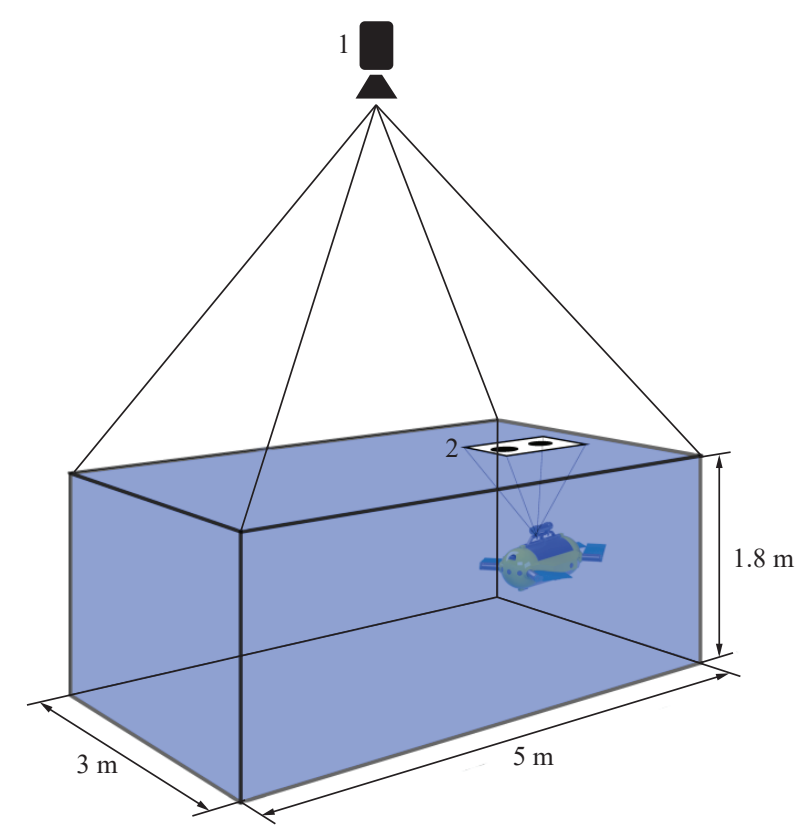

Fig. 6. Experimental setup for model identification and validation. Experiment was conducted in a $25 \mathrm{~m}$ swimming pool. The robot was mounted on a floating bar (2) which was tracked by the overhead camera (1).

in x-direction known from the fin characterization experiment and vehicles inertia matrix's first diagonal component $M_{x}^{R B}$ is equal to the vehicle's mass. The parameters $M_{x}^{A}$ and $D_{x}$ can be found using the least-squares method over the full experimental data set. We repeated the process with various different actuation parameters and selected the average of the found coefficients.

To validate the precision of the model we compared the experimentally measured motion and the model-predicted motion in each degree of freedom. The obtained results for a single actuation amplitude and frequency are presented on Fig. 7. It can be seen that the model-predicted position of the robot matches the actual position with a high precision.

\section{Control Architecture}

We propose a modular, easily extensible control architecture that allows rapid and simple controller implementation and testing, but which also can be used during a wide range of different real mission scenarios (see Fig 8). The control architecture is implemented fully in general matrix form. Matrix form implementation allows to quickly work with different DOFs without the need to reimplement any parts of the architecture if additional DOF's need to be added to the control. The second feature, which simplifies the testing and implementation, is 

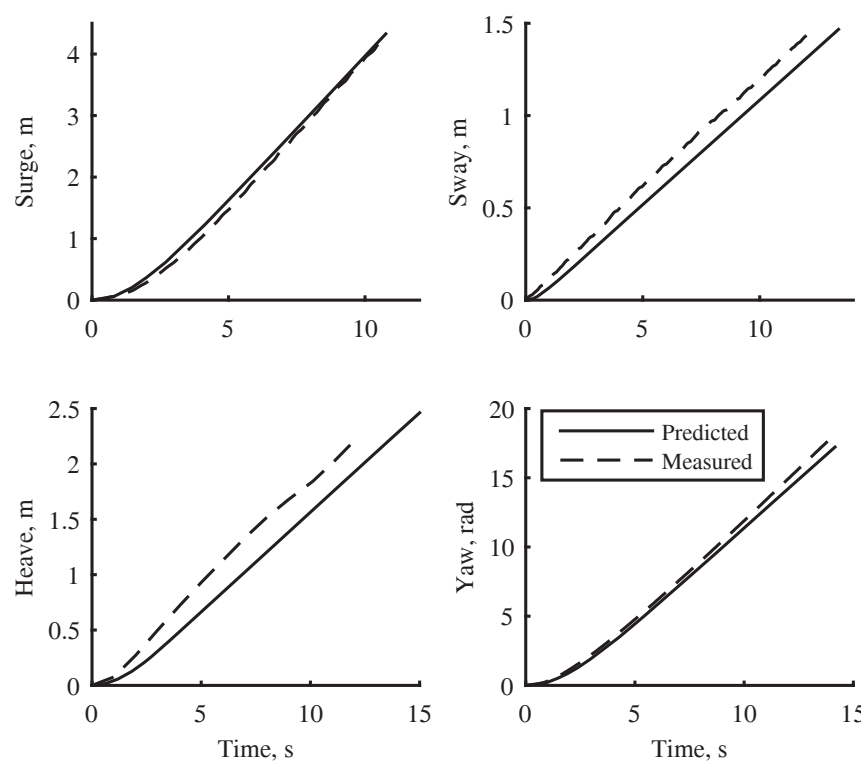

Fig. 7. Model validation for four main degrees of freedom. Plots show the model predicted (solid line) and experimentally measured (dashed line) position in different degrees of freedom when the fins are actuated at constant parameters (frequency of $2 \mathrm{~Hz}$ and amplitude of $50^{\circ}$ )

that the control architecture is composed of different modules, which all are implemented as independent ROS nodes. Each node can be easily replaced or modified for different scenarios. Also, additional nodes can be added for new control scenarios. In the current study the core of the control architecture is composed of the following nodes: Automatic Control for different DOFs, Dynamical Model, Priority Management, Force and Torque Calculation, Actuation, Odometry Estimation, Trajectory Management, and Human Intervention.

The general idea behind the proposed control is that each degree of freedom is separately and independently controlled by the best controller chosen for this DOF. The output of each controller is then prioritized using smooth functions, depending on the desired control action, to reduce the actuation coupling problem. The controllers can be individually tuned and replaced with any different control method or with manual inputs from the remote controller.

\section{A. Automatic control}

The automatic control node includes the controller implementations for each DOF. To choose the most suitable control approach we have experimentally tested different model-free and modelbased controllers. In section $\mathrm{V}$ we present the comparison of the following controllers: 


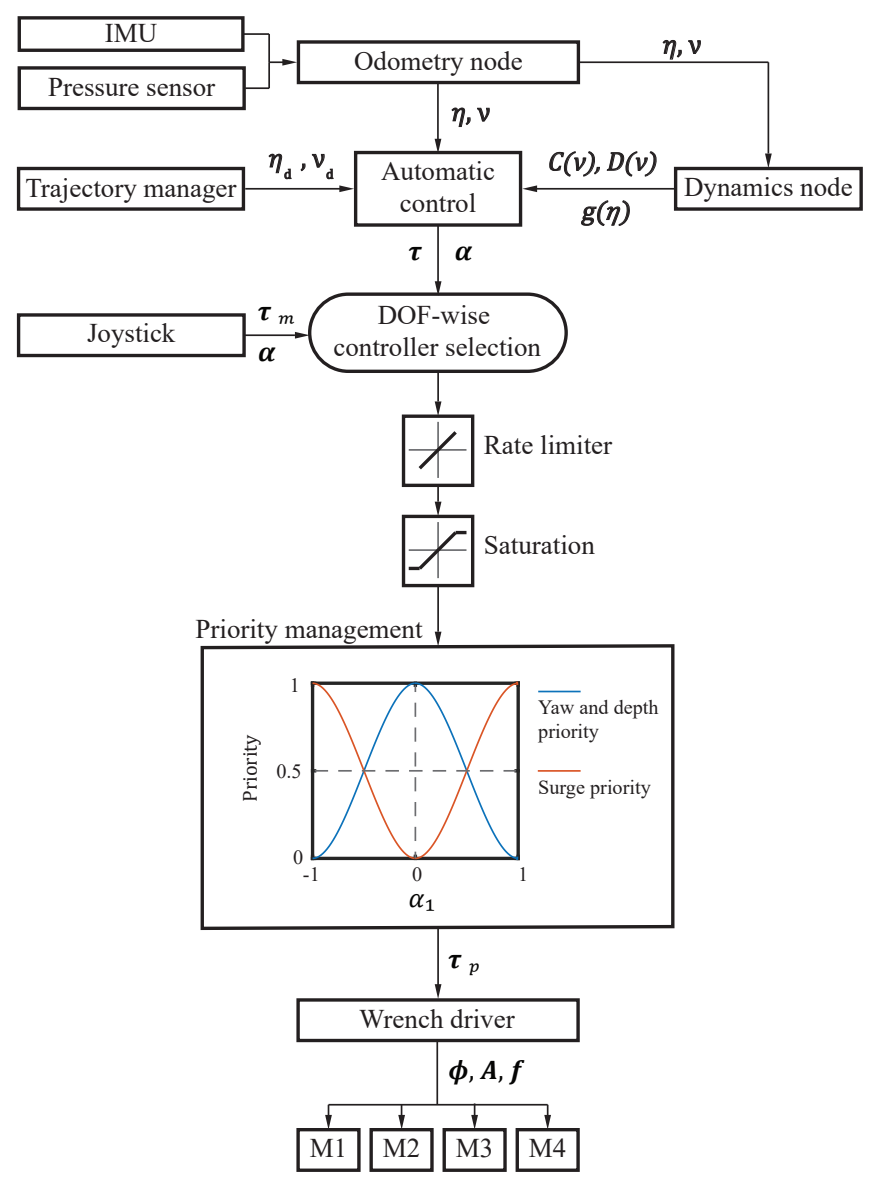

Fig. 8. Proposed motion control architecture

- PID

- Inverse dynamics (ID)

- Inverse dynamics with nonlinear feedback gains (IDNL)

- Adaptive inverse dynamics (AID)

\section{B. Human intervention}

In addition to the automatic control, all DOFs can be controlled manually using a joystick. Joystick outputs axis values between -1 to 1 . These values are proportionally mapped to manual control input $\tau_{m}$. For each DOF, operator can choose which control input the vehicle uses, manual or automatic. 


\section{Priority management}

The priority management node offers a solution to the DOF actuation coupling problem. Based on actuation redundancy, task priority control has been used in robotics to prioritize between different tasks [36] but as opposed to task priority control, DOF priority control is implemented to solve the problem of actuation coupling. DOF prioritization strategy works by assigning priorities to the control actions using smooth functions. To prioritize the DOFs, control input vector $\tau$ elements are multiplied by weight vector $\boldsymbol{p}=\left[p_{x}, p_{y}, p_{z}, p_{\varphi}, p_{\vartheta}, p_{\psi}\right]$ elements. The priority of each DOF varies from 0 to 1 . To assign priorities, different sets of laws could be created. Our tests show that on U-CAT it is best to use laws that assign priorities depending on the control action of a single DOF. We propose laws that give highest priority to yaw and depth control when surge action is small. When surge action increases, its priority increases and the priority of depth and yaw decreases. In principle, the control is modified depending on what motion the vehicle is about to perform. When the vehicle is close to its desired $x, y$ position, it can precisely hover and orient itself towards the desired direction. When a new waypoint is commanded or when the operator wants to move forward, surge action increases and the action of depth and yaw decreases. The laws we propose are the following:

$$
\begin{gathered}
p_{z}=p_{\psi}=c_{1}\left|\alpha_{x}\right|^{3}+c_{2}\left|\alpha_{x}\right|^{2}+c_{3}\left|\alpha_{x}\right|+c_{4} \\
p_{x}=1-p_{z}
\end{gathered}
$$

where $\alpha_{x}=\tau_{x} / \tau_{x \max }$ is the normalized control input. Constants $c_{i}$ are such that $0<\alpha_{1}<$ $1 \rightarrow 0<p_{z}<1$. The rules are plotted in priority management block of Fig. 8 .

\section{Wrench driver}

The wrench driver converts the generalized, priortized control input $\tau_{p}$ into fin actuation parameters $A_{i}, f_{i}$ and $\Phi_{i}$. It uses a set of simple rules to convert forces and moments of different DOFs to corresponding fin thrust forces $F_{i}$. These rules are implemented to achieve motions illustrated in Fig. 3. The conversion from fin thrust forces to actuation parameters is found from (3). We use a fixed frequency of $2 \mathrm{~Hz}$ and only vary the oscillation amplitudes $A_{i}$ and directions $\Phi_{i}$. Wrench driver forwards the actuation parameters to Maxon motor controllers who handle the position control of fins. 


\section{E. Odometry and trajectory}

Odometry node calculates the positions and velocities of the vehicle using the feedback from IMU and pressure sensor. It also estimates the position of the vehicle in the world-fixed frame using the dynamics of the vehicle. However, we do not use this estimation in the current study due to the lack of a good state observer.

The trajectory manager creates smooth position and velocity trajectories. In the current study it uses predefined timed setpoints written in a file. It then uses hyperbolic tangent functions to calculate smooth transitions between setpoints. The trajectory manager is also able to read setpoints from other sources. For example, a remote controller can be used to modify the desired depth and yaw.

\section{2-DOF CONTROL}

The Automatic Control Unit in Fig. 8 is responsible for automatically controlling the specified degrees of freedom. This control unit can work in combination with the manual control by joystick, leaving the control of some DOFs for a human operator. An automatic control of some DOFs would take some cognitive load from the human operator and let the operator concentrate on better completing the misson. A typical scenario may be a human operation guiding the vehicle along a desired trajectory by visual feedback on $x-y$ plane while the depth and yaw of the vehicle are automatically controlled. In order to select the most suitable controller for automatic control, we propose here the implementation and comparative experiments of four different feedback controllers (one non-model-based and three model-based controllers) at 3 different implementation scenarios with a varying degree of complexity. All the proposed controllers are tested and compared at the following scenarios:

- Nominal case in a controlled environment

- External disturbance rejection

- Robustness towards uncertainties

\section{A. Controllers}

1) Proportional Integral Derivative (PID): The PID is a well known and well established non-model-based controller [37]. The control law can be expressed by:

$$
\tau=K_{P} \tilde{\eta}+K_{I} \int_{0}^{t} \tilde{\eta} d t+K_{D} \dot{\tilde{\eta}}
$$


$\tilde{\eta}=\eta-\eta_{d}$ is the tracking error, and $\dot{\tilde{\eta}}$ is its first time derivative. $K_{P}, K_{I}, K_{D}$ are positive definite matrices representing the proportional, the integral, and the derivative feedback gains respectively.

2) Inverse dynamics (ID): The main idea of this controller is to fully linearize the dynamics of the robot based on a nonlinear feedback law to obtain a linear system [33]. Consider the nonlinear dynamics (1) of the robot, and consider the following nonlinear feedback control law:

$$
\tau=M a^{b}+n(\nu, \eta)
$$

with $\boldsymbol{a}^{\boldsymbol{b}}$ the body frame commanded acceleration, that can be calculated from a simple transformation between the body and the earth fixed-frames as $\boldsymbol{a}^{\boldsymbol{b}}=J^{-1}\left(\boldsymbol{a}^{\boldsymbol{n}}-\dot{J} \boldsymbol{\nu}\right)$, and $\boldsymbol{a}^{\boldsymbol{n}}=$ $\ddot{\eta}_{d}-K_{P} \tilde{\eta}-K_{I} \int_{0}^{t} \tilde{\eta} d t-K_{D} \dot{\tilde{\eta}}$, where $\eta_{d}$ is the desired trajectory and $\ddot{\eta}_{d}$ is its corresponding acceleration. The control law (7), if replaced in the robot's dynamics (1), leads to:

$$
(J \dot{\nu}+\dot{J} \nu)=\ddot{\eta}_{d}-K_{P} \tilde{\eta}-K_{I} \int_{0}^{t} \tilde{\eta} d t-K_{D} \dot{\tilde{\eta}}
$$

Now consider the robot's kinematics (2), its first derivative leads to $\ddot{\eta}=\dot{\boldsymbol{J}} \boldsymbol{\nu}+\boldsymbol{J} \dot{\boldsymbol{\nu}}$, which if combined with (8) gives:

$$
\ddot{\tilde{\eta}}+K_{D} \dot{\tilde{\eta}}+K_{P} \tilde{\eta}+K_{I} \int_{0}^{t} \tilde{\eta} d t=0
$$

With an appropriate choice of the feedback gains $K_{P}, K_{I}, K_{D}$, the closed-loop dynamics is asymptotically stable.

3) Inverse dynamics with nonlinear feedback gains (IDNL): This approach has the same control law as the previous one, except that feedback gains are no longer constants. The use of nonlinear varying feedback gains may improve the tracking performance of the controller. For instance, the Nonlinear Computed Torque [38] and the Nonlinear Augmented PD [39] provided better tracking performance than the conventional computed torque and augmented PD controllers respectively. For our case, let's consider the following nonlinear proportional gain $K_{P}(\cdot)$ :

$$
\boldsymbol{K}_{\boldsymbol{P}}(e)= \begin{cases}k_{p_{0}}|e|^{\alpha_{1}-1}, & |e|>\delta_{1}, \\ k_{p_{0}} \delta_{1}^{\alpha_{1}-1}, & |e| \leqslant \delta_{1} .\end{cases}
$$

where $k_{p_{0}}$ is its maximum values, $\alpha_{1}$ is the nonlinearity decreasing parameter and $\delta_{1}$ its activation threshold (as illustrated in Fig. 9). The derivative gain $K_{D}(\cdot)$, also illustrated in Fig. 9, may be expressed by:

$$
K_{\boldsymbol{D}}(\dot{e})= \begin{cases}k_{d_{0}}|\dot{e}|^{\alpha_{2}-1}, & |\dot{e}|>\delta_{2}, \\ k_{d_{0}} \delta_{2}^{\alpha_{2}-1}, & |\dot{e}| \leqslant \delta_{2}\end{cases}
$$




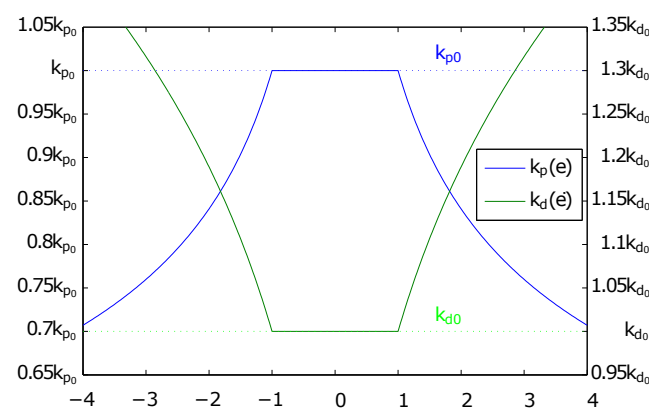

Fig. 9. Typical evolution of the nonlinear proportional $K_{P}$ and derivative $K_{D}$ gain versus position error $e$ and velocity error $\dot{e}$ (respectively) with $\alpha_{1}=0.75, \delta_{1}=1, \alpha_{2}=1.25$ and $\delta_{2}=1$.

with $k_{d_{0}}$ is its minimum value, $\alpha_{2}$ is the nonlinearity increasing parameter and $\delta_{2}$ its activation threshold.

4) Adaptive inverse dynamics (AID): The adaptive inverse dynamics controller is a state feedback controller with an adaptation control term. It provides an online estimation of possible unknown model parameters in order to ensure a good trajectory tracking [33]. The control law can be expressed by:

$$
\tau=\hat{M} a^{b}+\hat{n}(\nu, \eta)
$$

where the hatted variables denote the dynamics matrices based on estimations, $\hat{n}(\nu, \eta)$ the estimate of $n(\nu, \eta)$ in (7). Since the dynamic model is linear in its parameters, the adaptive control law (12) can then be rewritten as:

$$
\tau=\Phi\left(a^{b}, \nu, \eta\right) \hat{\theta}
$$

where $\boldsymbol{\Phi}$ represents the regressor and $\hat{\boldsymbol{\theta}}$ is the vector of the online estimated parameters. $\boldsymbol{a}^{\boldsymbol{b}}$ and $\boldsymbol{a}^{\boldsymbol{n}}$ are computed as in the inverse dynamics controller. The vector of estimated parameters is updated online based on the following adaptive law:

$$
\dot{\hat{\theta}}=-\Gamma \Phi^{T}\left(a^{b}, \nu, \eta\right) J^{-1} y
$$

where the diagonal positive definite matrix $\Gamma$ represents the adaptation gain, $J$ the transformation matrix, and $\boldsymbol{y}$ is the combined tracking error given by $\boldsymbol{y}=c_{0} \tilde{\boldsymbol{\eta}}+c_{1} \dot{\tilde{\boldsymbol{\eta}}} \cdot c_{0}$ and $c_{1}$ are positive constant gains, that may be chosen using the algorithm presented in [33], and based on Barbalat's lemma, it ensures that $\boldsymbol{y}$ converges to zero. 


\section{B. Implementation details}

The comparative experiments of 2-DOF control have been carried out in the swimming pool of the University of Montpellier in France with the aim of choosing the most suitable controller for the automatic control mode of the architecture of Fig. 8. The 2DOF control experiments where implemented as follows:

- The parameters of each controller are tuned for the nominal case and kept unchanged for the other scenarios.

- All the proposed controllers were implemented on U-CAT using the ROS based control architecture presented in section IV

- Two degrees of freedom are to be simultaneously controlled (namely depth and yaw).

- All the relevant real-time data were saved to ROS log files (rosbags). The use of rosbags enables inspecting the data exactly in the same form as used by the robot software.

Next we will briefly describe all the three implementation scenarios, present the results and discuss the selection of the 2-DOF controller. The tuned parameters of the proposed controllers are summarized in Tables I, II, III and IV.

TABLE I

SUMMARY OF THE PARAMETERS OF THE PID CONTROLLER.

\begin{tabular}{|c|c|c|c|}
\hline Parameter & $\boldsymbol{K}_{\boldsymbol{P}}$ & $\boldsymbol{K}_{\boldsymbol{I}}$ & $\boldsymbol{K}_{\boldsymbol{D}}$ \\
\hline Value & $\left(\begin{array}{cc}200 & 0 \\
0 & 8\end{array}\right)$ & $\left(\begin{array}{cc}7.5 & 0 \\
0 & 0.05\end{array}\right)$ & $\left(\begin{array}{cc}80 & 0 \\
0 & 6\end{array}\right)$ \\
\hline
\end{tabular}

TABLE II

SUMMARY OF THE PARAMETERS OF THE ID CONTROLLER.

\begin{tabular}{|c|c|c|c|}
\hline Parameter & $\boldsymbol{K}_{\boldsymbol{P}}$ & $\boldsymbol{K}_{\boldsymbol{I}}$ & $\boldsymbol{K}_{\boldsymbol{D}}$ \\
\hline Value & $\left(\begin{array}{cc}0.6 & 0 \\
0 & 2.5\end{array}\right)$ & $\left(\begin{array}{cc}0.075 & 0 \\
0 & 0.1\end{array}\right)$ & $\left(\begin{array}{cc}0.2 & 0 \\
0 & 2.2\end{array}\right)$ \\
\hline
\end{tabular}


TABLE III

SUMMARY OF THE PARAMETERS OF THE IDNL CONTROLLER.

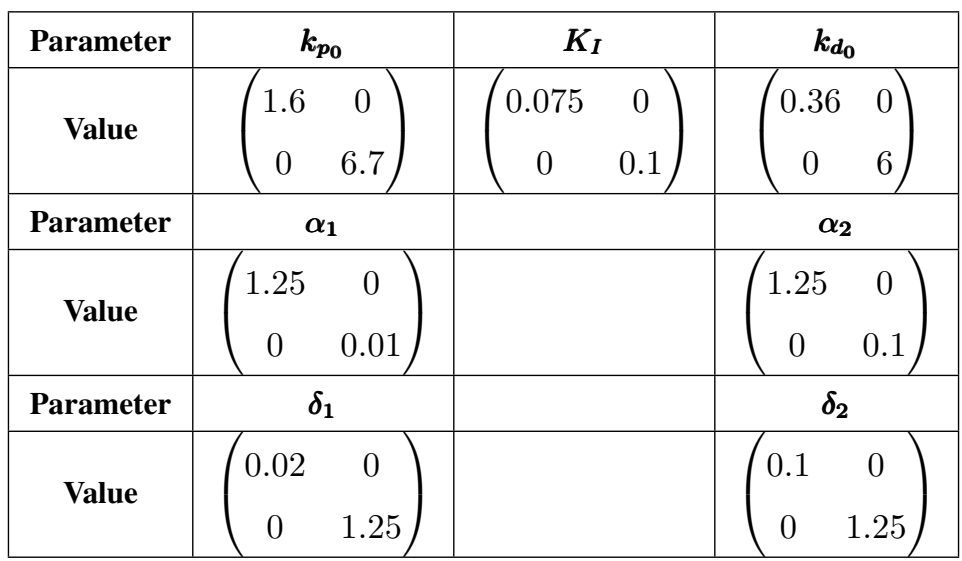

TABLE IV

SUMMARY OF THE PARAMETERS OF THE AID CONTROLLER.

\begin{tabular}{|c|c|c|c|}
\hline Parameter & $K_{\boldsymbol{P}}$ & $K_{\boldsymbol{I}}$ & $K_{\boldsymbol{D}}$ \\
\hline Value & $\left(\begin{array}{cc}0.6 & 0 \\
0 & 2.5\end{array}\right)$ & $\left(\begin{array}{cc}0.075 & 0 \\
0 & 0.1\end{array}\right)$ & $\left(\begin{array}{cc}0.2 & 0 \\
0 & 2.2\end{array}\right)$ \\
\hline Parameter & $\boldsymbol{c}_{\boldsymbol{0}}$ & $\boldsymbol{c}_{\boldsymbol{1}}$ & $\boldsymbol{\Gamma}$ \\
\hline Value & $\left(\begin{array}{cc}0.1 & 0 \\
0 & 0.1\end{array}\right)$ & $\left(\begin{array}{cc}0.075 & 0 \\
0 & 0.075\end{array}\right)$ & $\left(\begin{array}{cc}1.5 & 0 \\
0 & 3\end{array}\right)$ \\
\hline
\end{tabular}

\section{Experimental scenarios}

1) Experimental scenario 1 - Nominal case: We first test all the proposed control solutions in the nominal case. This means that the tests will be conducted without external disturbances and without changes in the dynamic parameters of the robot. The main objective is to track predefined time-varying reference trajectories along both controllable degrees of freedom (depth and yaw).

2) Experimental scenario 2 - External disturbance rejection: The main objective behind this experimental scenario is to test the ability of the proposed controllers to reject external disturbances. The same predefined time-varying reference trajectories, used for the previous scenario, are tracked. Once the system outputs (depth and yaw) reach the steady-state value, an 


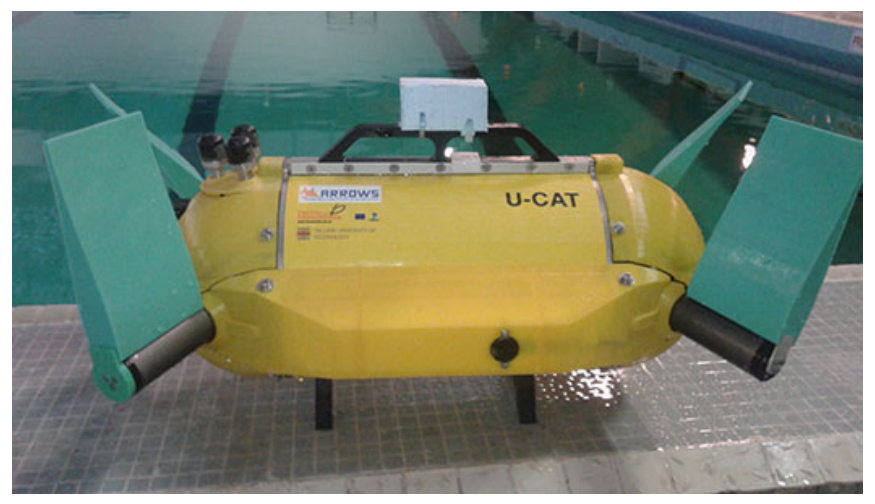

Fig. 10. View of the robot with increased floatability, a piece of float fixed to the body of the robot increases its buoyancy.

external disturbance is applied, the behaviour of the system is recorded to show the reaction of the controller and its ability to steer back the system's outputs to their reference values.

3) Experimental scenario 3 - Robustness towards uncertainties: With this scenario we test the robustness of the controllers towards parameters' uncertainties. We change one of the dynamic parameters of the robot (namely the buoyancy), and check how well the proposed controllers are able to compensate for such change with respect to the nominal case. It is worth to emphasize that all the parameters of the proposed controllers are kept unchanged. Fig. 10 illustrates how the buoyancy of the robot was changed: a piece of a float was fixed on the body of the robot, which will increase its buoyancy. The proposed experimental scenario consists of tracking reference trajectories while the buoyancy of the robot is increased.

\section{Obtained results}

The experimental results are plotted in Fig. 11 (PID, ID and IDNL controllers) and Fig. 12 (AID controller). The plots show the desired and actual depth and yaw during each experiment. To compare the results, we use the root-mean-square tracking error (RMS) calculated over the full length of each experiment (from diving to re-surfacing). In addition to the RMS error of depth $\left(R M S_{\text {depth }}\right)$ and $\operatorname{yaw}\left(R M S_{\text {yaw }}\right)$ we use the mean of these two values $(\overline{R M S})$ to describe the combined performance of the whole system. All the depth and yaw RMS values are shown in Table $\mathrm{V}$ and all the mean RMS values are shown in Table VI.

It is important to note that RMS values are comparable only in the Scenario 1 - Nominal case and Scenario 3 - Robustness towards uncertainties. RMS value does not give meaningful information in Scenario 2 - External disturbance rejection, as the applied external disturbance 

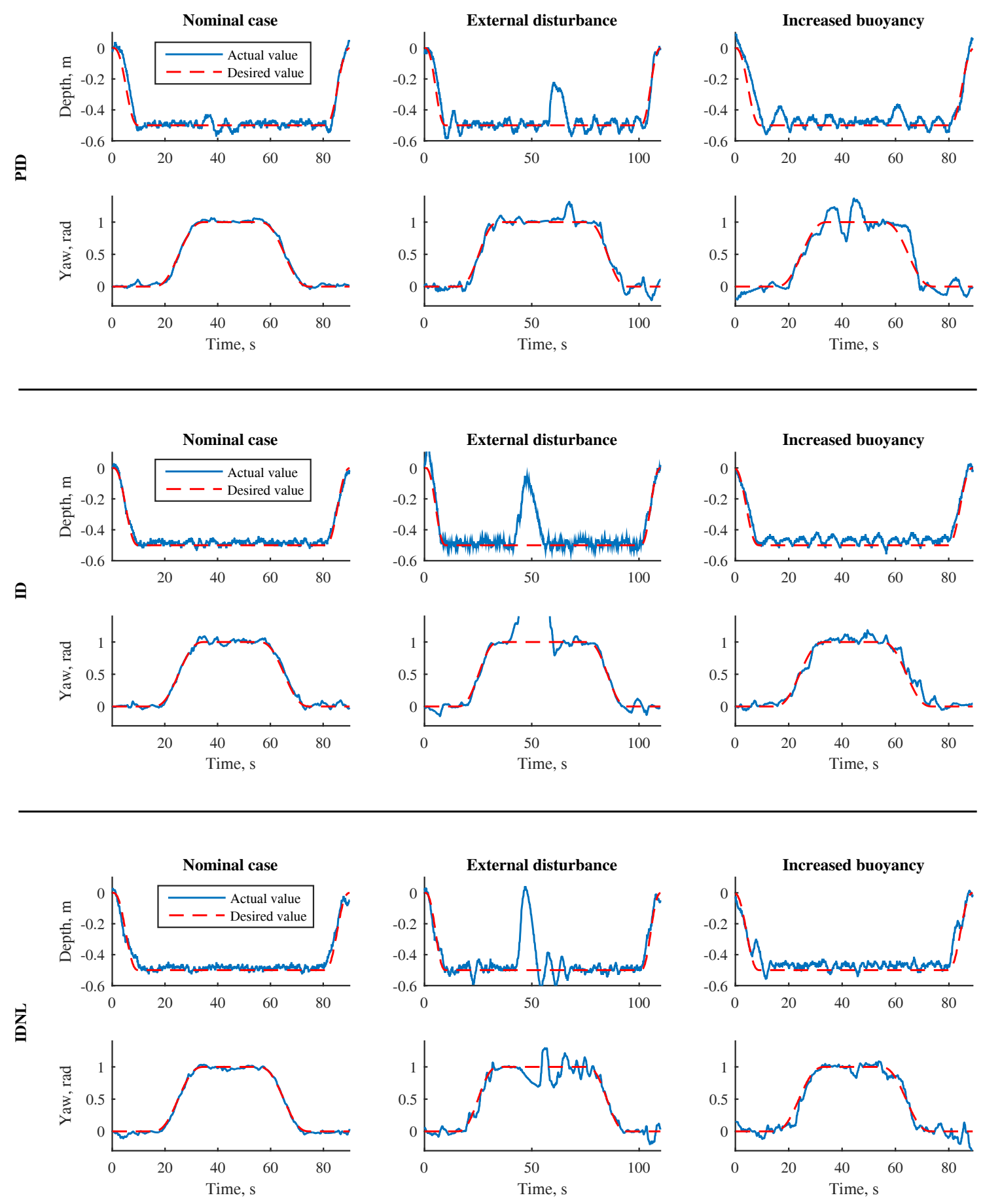

Fig. 11. 2DOF controller comparison results in three different test scenarios. Part 1 - PID, inverse dynamics and inverse dynamics with nonlinear feedback gains 

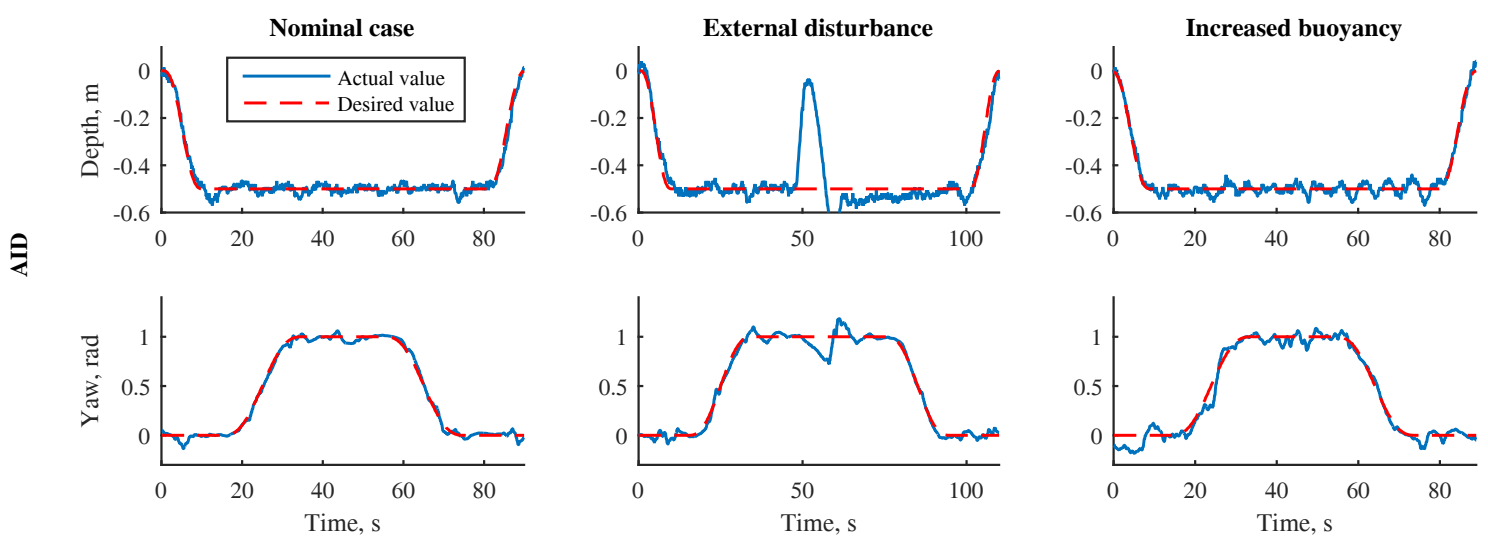

Fig. 12. 2DOF controller comparison results in three different test scenarios. Part 2 - Adaptive inverse dynamics controller

was not equal in all the experiments. In this scenario we qualitatively estimate how well does the vehicle return to a steady state after the disturbance.

The results show that in the nominal case, all four proposed controllers hold the vehicle on the predefined trajectory without any major deviations or oscillations. However, the RMS values differ significantly. As expected, the mean RMS of PID is bigger than that of the model based controllers in both the nominal and the increased buoyancy scenario. In the case of increased buoyancy the PID is not able to correctly track the yaw trajectory due to the instability in depth control and the coupled degrees of freedom.

The ID depth controller performs $33.9 \%$ better than PID in nominal case and $29.4 \%$ better than PID in case of increased buoyancy. Even though the yaw performance in nominal case was slightly lower than that of the PID, the combined error of ID was $7.7 \%$ smaller in nominal case and $40.0 \%$ smaller in the case of modified buoyancy. The external disturbance experiment shows that the ID controller is very well able to return the vehicle to the desired values after disturbance. There are no oscillations in the depth and yaw values and only slight overshoot in the yaw.

Contrary to our expectations, IDNL does not perform better than ID. Even though its combined performance in the nominal case is $1.6 \%$ better than that of the ID, the mean RMS in the modified buoyancy case is $17.9 \%$ higher that of the ID (28.7\% bigger depth RMS and $12.2 \%$ bigger yaw RMS). Also, the external disturbance experiment reveals strong oscillations in depth and failure to return to desired yaw value after disturbance.

The AID's mean RMS is $9.3 \%$ smaller than that of the ID in nominal case and $21.7 \%$ smaller 
TABLE V

RMS VALUES FOR DEPTH / YAW

\begin{tabular}{c||c|c}
\hline & $\begin{array}{c}\text { Scenario 1 } \\
\text { depth } \mid \text { yaw }\end{array}$ & $\begin{array}{c}\text { Scenario 3 } \\
\text { depth } \mid \text { yaw }\end{array}$ \\
\hline \hline PID & $34.5 \mathrm{~mm} \mid 30.3 \mathrm{mrad}$ & $56.1 \mathrm{~mm} \mid 136.6 \mathrm{mrad}$ \\
\hline ID & $22.8 \mathrm{~mm} \mid 37.0 \mathrm{mrad}$ & $39.6 \mathrm{~mm} \mid 76.0 \mathrm{mrad}$ \\
\hline IDNL & $29.6 \mathrm{~mm} \mid 29.2 \mathrm{mrad}$ & $51.0 \mathrm{~mm} \mid 85.3 \mathrm{mrad}$ \\
\hline AID & $21.2 \mathrm{~mm} \mid 33.0 \mathrm{mrad}$ & $22.2 \mathrm{~mm} \mid 68.3 \mathrm{mrad}$ \\
\hline
\end{tabular}

TABLE VI

MEAN RMS VALUES

\begin{tabular}{c||c|c}
\hline & Scenario 1 & Scenario 3 \\
\hline \hline PID & 32.4 & 96.3 \\
\hline ID & 29.9 & 57.8 \\
\hline IDNL & 29.4 & 68.1 \\
\hline AID & 27.1 & 45.2 \\
\hline
\end{tabular}

than that of the ID in the case of modified buoyancy. Based on these values AID has the best performance in the nominal case and in the case of modified buoyancy (16\% better than PID in nominal case and 53\% better than PID in the case of modified buoyancy). However, the external disturbance experiment reveals one disadvantage of the adaptive controller. After the disturbance, the controller needs approximately $40 \mathrm{~s}$ to take the vehicle back to the desired depth. During these $40 \mathrm{~s}$ there is a steady depth error slowly converging towards zero. The reason for this error is that as a result of a strong external disturbance the adaptive model parameters diverge from their desired values and it takes time until the parameters re-adapt to their correct values.

Based on the controller comparison we can say that PID and ID are the only two controllers that show good results in all the three experimental scenarios. However, due to the compensation of the nonlinear components of the vehicle dynamics, the ID controller performs significantly better. IDNL fails to perform well in our case probably because of a more difficult and less intuitive parameter tuning process. Since we are looking for robust and easily applicable control 
methods, IDNL is not the best approach. As expected, AID performs significantly better than any other controller in the test of robustness towards model uncertainties. Therefore, this controller would be the best choice for missions where the vehicle dynamics changes during the operation. This includes for example the case where the robot is operated on a long tether that affects the vehicle's dynamics. However, AID is not appropriate for missions in which the vehicle may be exposed to strong external disturbances, such as collision with underwater objects. Our control architecture allows seamless switching between different control approaches even during the mission. Either ID or AID can be chosen by the operator depending on the situation.

\section{3-DOF CONTROL}

This section experimentally validates the proposed control architecture with DOF prioritization for $3 \mathrm{D}$ motion of the robot. We describe 5 control strategies where some of the degrees are controlled by the operator with the joystick and others automatically while using the DOF priority manager illustrated in Fig. 8. These tests are also compared to the scenario where the DOF priority management is switched off.

The experiments were conducted in a $60 \mathrm{~m}$ long, $5 \mathrm{~m}$ wide and $3 \mathrm{~m}$ deep fresh water tow tank of Tallinn University of Technology. In all the experiments the robot followed a lawnmower trajectory at constant depth of $1 \mathrm{~m}$. Each experiment started with a dive and ended with a resurface. We chose the lawnmower trajectory because of its wide use in underwater inspection tasks. During the experiments the robot recorded its depth and yaw data into ROS log files. As the acoustic positioning methods did not work in a concrete tank, we did not have any real-time position feedback for translational motions on the horizontal plane. However, we used a calibrated overhead camera for recording the vehicle trajectories. After the experiments we manually tracked the position of U-CAT from the overhead video frame by frame.

\section{A. Experimental scenarios}

We tested the following 5 control approaches:

1) Fully manual control: An experienced U-CAT operator controlled depth, yaw and surge to follow the predefined trajectory as precisely as possible while keeping constant depth. In this scenario the automatic control unit on Fig. 8 is switched off and the commands from the joystick are processed by the priority manager. The operator used visual feedback and a depth reading on screen. The lawnmower trajectory was relatively loosely defined in our experiments, as there 
was no precise position feedback other than visual. The operator used markers on the poolside to approximately travel a same distance with every short leg of the trajectory. For comparative reasons the operator also tried to follow the same trajectory timing as in other experiments.

2) Depth autopilot: In this experiment the automatic control unit in Fig. 8 controls only depth while the operator controlled other DOFs (yaw and surge) manually.

3) Depth and yaw autopilot: In this experiment the operator's manual control was kept minimal as he only controlled the surge motion with the joystick to move the vehicle from one turning point to another while depth and yaw motion where controlled automatically.

4) Fully autonomous control: As U-CAT is designed to eventually be fully autonomous, we also tested the extensibility of our control approach to fully autonomous control mode. In this case the vehicle was untethered and the operator was removed from the control loop. The manual surge regulation was replaced by automatic open-loop control. As we did not have any surge velocity or position feedback, the surge control was implemented as a smooth predefined force trajectory.

5) Fully autonomous control without DOF prioritization: In this experiment we repeated the fully autonomous control scenario, but we disabled the priority manager. All 3 DOF (depth, yaw and surge) where controlled by the Autonomous control unit in Fig. 8 while the Priority Manager was turned off.

\section{B. Obtained Results}

The desired and actual trajectories for each of the above experiments are shown in Fig. 13. Root mean square (RMS) error values calculated over the full length of experiments are marked on the graphs.

1) Manual Control: Fig. 13a shows the desired and actual trajectories of depth, yaw, $\mathrm{x}$ and y. From the figure it can be easily seen that the trajectory following precision is poor (depth RMS error of $27.6 \mathrm{~cm}$ and yaw RMS error of $39.8^{\circ}$ ). This illustrates the definite need for at least some autopiloting.

2) Depth Autopilot: In this experiment the depth autopilot was enabled while the operator manually controlled the surge and yaw. Results show $81 \%$ decrease in depth error compared to the manual control. In addition yaw error decreased $28 \%$ as now the operator had to concentrate on controlling only 2 DOFs instead of 3 . However, the overhead camera image still reveals the lack of precision in tracking straight lines. The vehicle turns rapidly. This kind of unsteady motion 

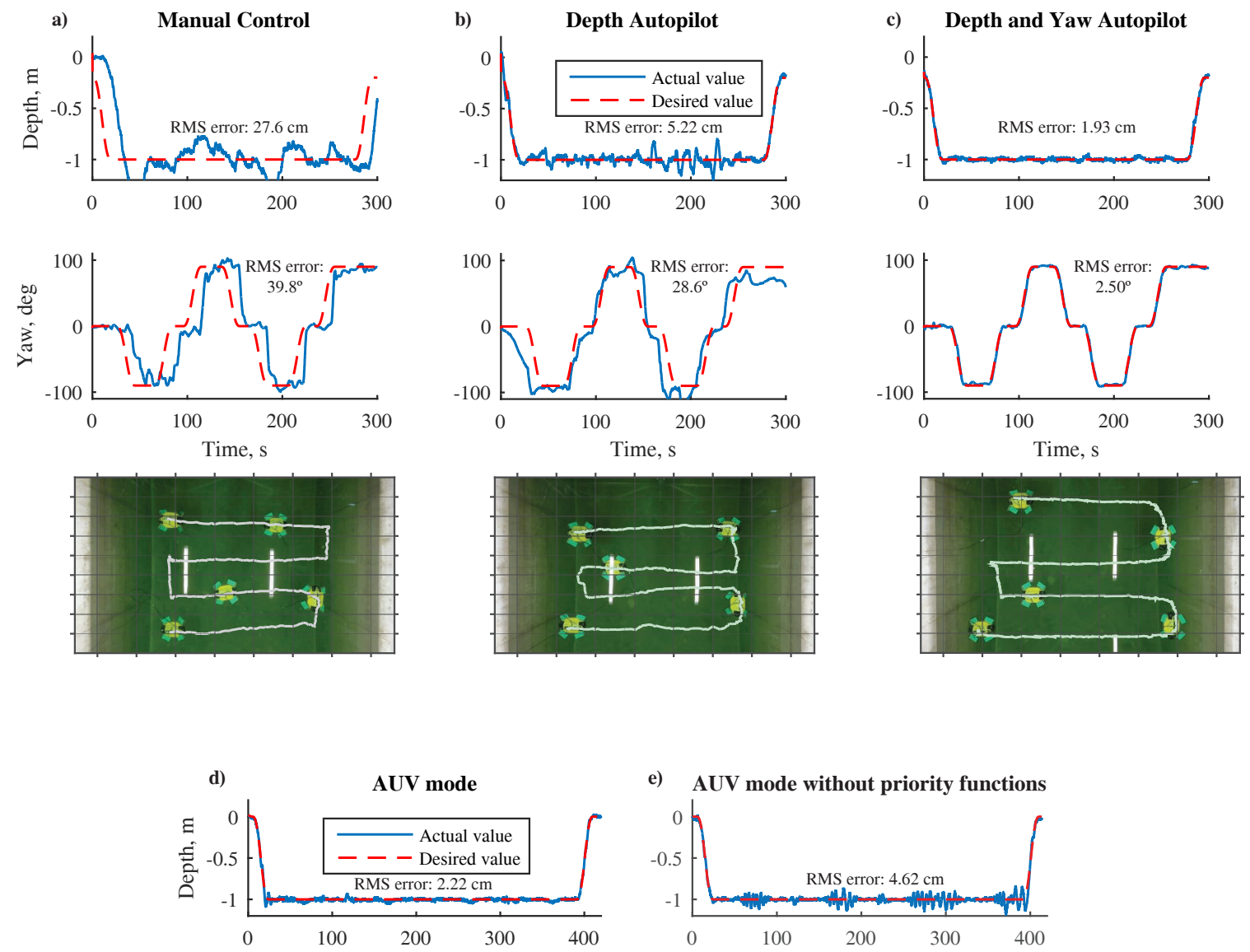

e) AUV mode without priority functions
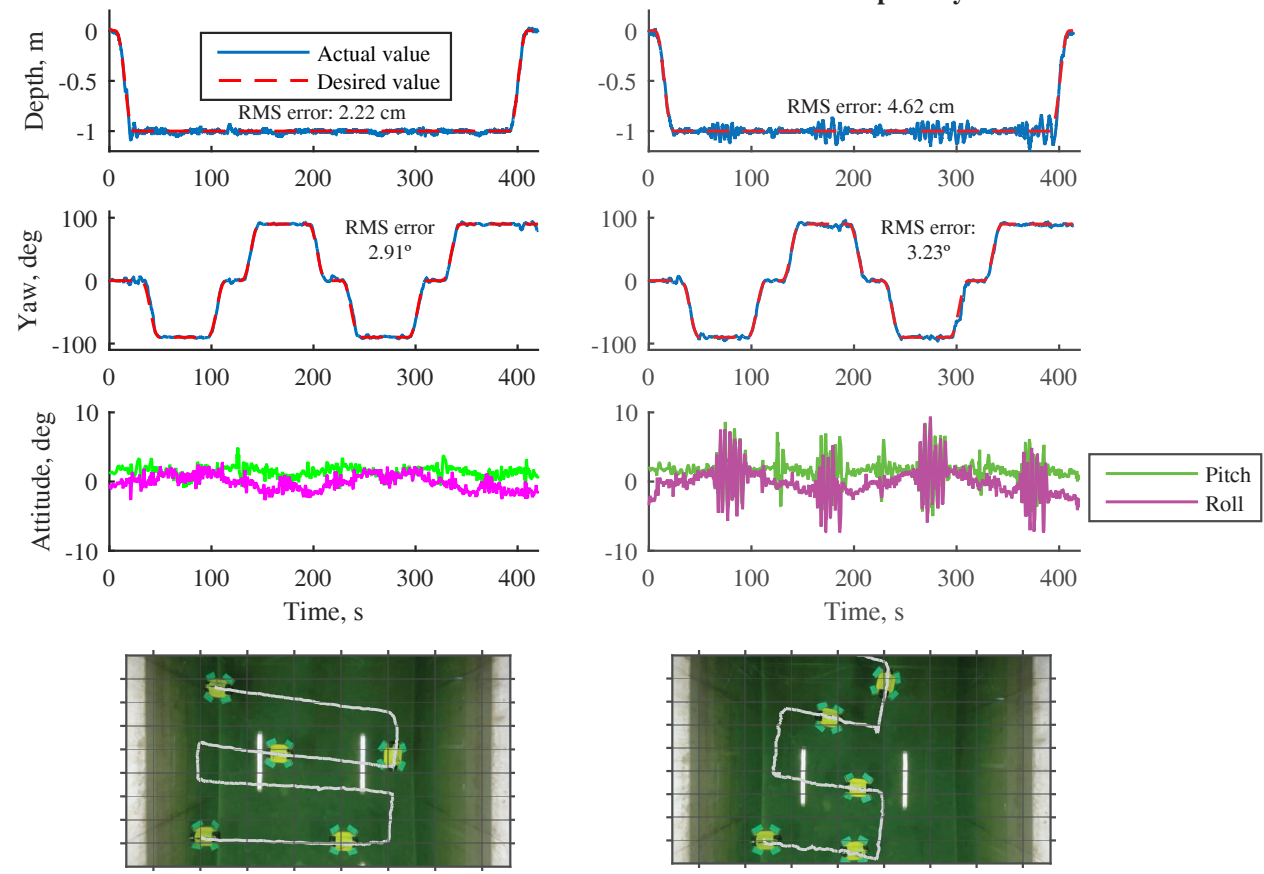

Fig. 13. Experimental results for 3 DOF control in 5 different control scenarios. 
would probably cause problems in video inspection tasks, especially when using automatic video processing methods such as mosaicking.

3) Depth and Yaw Autopilot: In this experiment we also enabled automatic yaw control. This time the operator intervention was minimal. He only had to control the surge motion to move the vehicle from one turning point to another. The obtained results (Fig. 13c) show high precision in yaw tracking (RMS error of $2.5^{\circ}$; improvement of $91 \%$ ). Also, due to more steady yaw the depth tracking error decreased by $63 \%$ compared to the scenario with only depth autopilot. More stable yaw is also visible from the overhead camera image. It shows how the robot moves in straight lines between turning points. Unfortunately, the short legs of the trajectory (vertical on the overhead camera images) are not perpendicular to the long legs. This is caused by the disturbance of the tether, which slowly pulled the vehicle sideways. The disturbances are inevitable and during ROV missions they have to be manually compensated for. The compensation can be relatively easily done as the U-CAT's fins also allow to actuate the sway motion to move the vehicle sideways. In this article, however, we do not consider sway control.

4) Fully Autonomous Control: The results depicted in Fig. 13d show that removing human intervention did not significantly reduce the tracking precision. Also, the tether disturbance is not any more visible on the overhead image. However, the figure reveals an IMU drift in yaw signal which was not present in our previous experiments. We have noticed that large IMU drift sometimes occurs due to electromagnetic noise emitted by the motor controllers. The drift is small enough $(0.9 \mathrm{deg} / \mathrm{min})$ to not affect the controller behaviour. For real missions the drift will be reduced by minimizing electromagnetic noise and by using magnetometers and Extended Kalman Filter. Despite the IMU issue the experiment shows that our control approach is suitable for simultaneously controlling 3 DOFs of the fully autonomous 4-fin vehicle. Once the vehicle has a reliable position or surge velocity estimation, the current surge controller can be replaced by a suitable closed-loop controller.

5) Fully autonomous control without DOF prioritization: The test results without the priority manager in Fig. 13 e show strong oscillations of depth value. The oscillations occur when the surge action increases and the vehicle tries to move forward. The figure also displays the roll and pitch angles in comparison to those during the previous experiment (Fig. $13 \mathrm{~d}$ ). It can be seen that the vehicle is strongly oscillating in pitch and roll. The oscillations are the result of a coupling between the actuation of different DOFs. All the controllers try to stabilize the vehicle, 
however the fin configuration does not allow to equally control all the DOFs at the same time. The resulting lawnmower trajectory is also different from that of the experiment 4 as the vehicle can not correctly output the predefined surge force. These results clearly show the importance of DOF prioritization in our system.

\section{VALIDATION IN NATURAL ENVIRONMENT}

This section describes two real-life test scenarios in natural environments where the control of the robot was validated during a real mission.

\section{A. ROV Inspection Missions of Submerged Machinery}

The first testsite is located in Rummu quarry in Estonia (59 $\left.13^{\prime} 35.8^{\prime \prime} \mathrm{N} 24^{\circ} 13^{\prime} 30.48^{\prime \prime} \mathrm{E}\right)$. Rummu quarry is an abandoned flooded limestone mine containing submerged building and several large mining machines. We inspected a mining excavator in $11 \mathrm{~m}$ depth with the U-CAT in an ROV mode. Two missions where carried out on this test site in different environmental conditions:

- 1 - Winter experiment (3rd of March 2016) - we launched the vehicle through an 1x1 meter hole (see Fig. 15a) cut into $20 \mathrm{~cm}$ thick layer of ice.

- 2 - Summer experiment (14th of July 2016) - we launched the vehicle from a small (4.3 m) rowboat.

During the mission the depth and yaw were autopiloted by the inverse dynamics controller. The operator used a joystick to modify the controller setpoints and to manually control the surge speed of the vehicle. He used the live video feedback (Fig. 15c) from the downward looking on-board camera to inspect the different parts of the machine.

Fig. 14 shows a typical test data of the yaw and depth position and the surge action during one of the dives. It can be seen that the autopilot is able to track the desired trajectory throughout the whole mission (depth RMS error of $8.6 \mathrm{~cm}$; yaw RMS error of $5.8^{\circ}$ ). According the operators' feedback the vehicle was relatively simple to control. He successfully gathered large amounts of video data of the machine during 2 missions.

Even though the tracking precision was high enough for ROV inspection, the combined RMS error is higher than in the controlled environment (depth RMS error of $1.9 \mathrm{~cm}$; yaw RMS error of $2.5^{\circ}$ ). This is mostly due to following factors: i) much longer tether; ii) environmental disturbing factors such as waves and currents; iii) in this experiment the operator modified the set values 

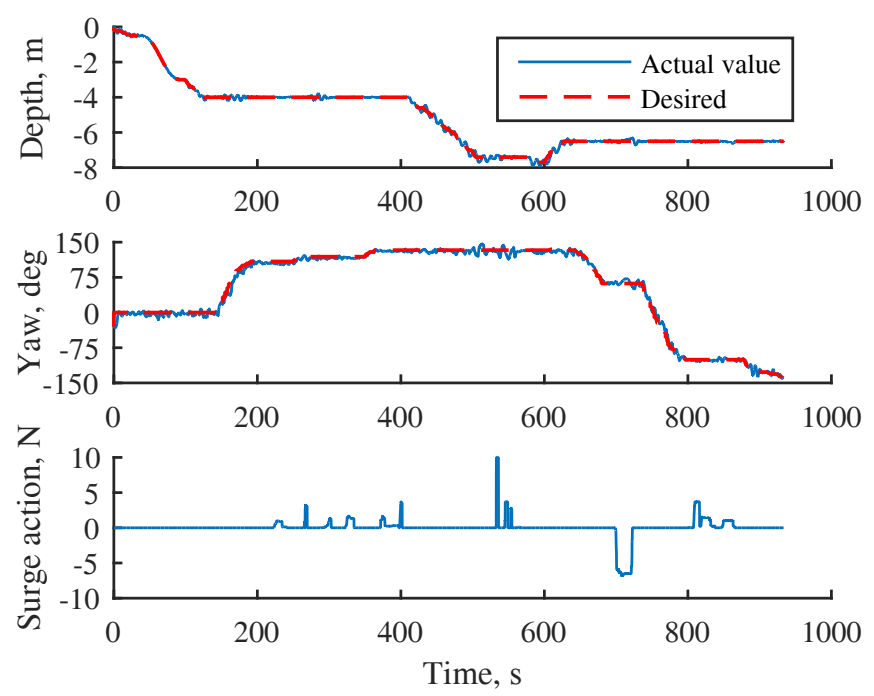

Fig. 14. Sample of position tracking and surge action data during the inspection mission done from the boat in Rummu quarry.

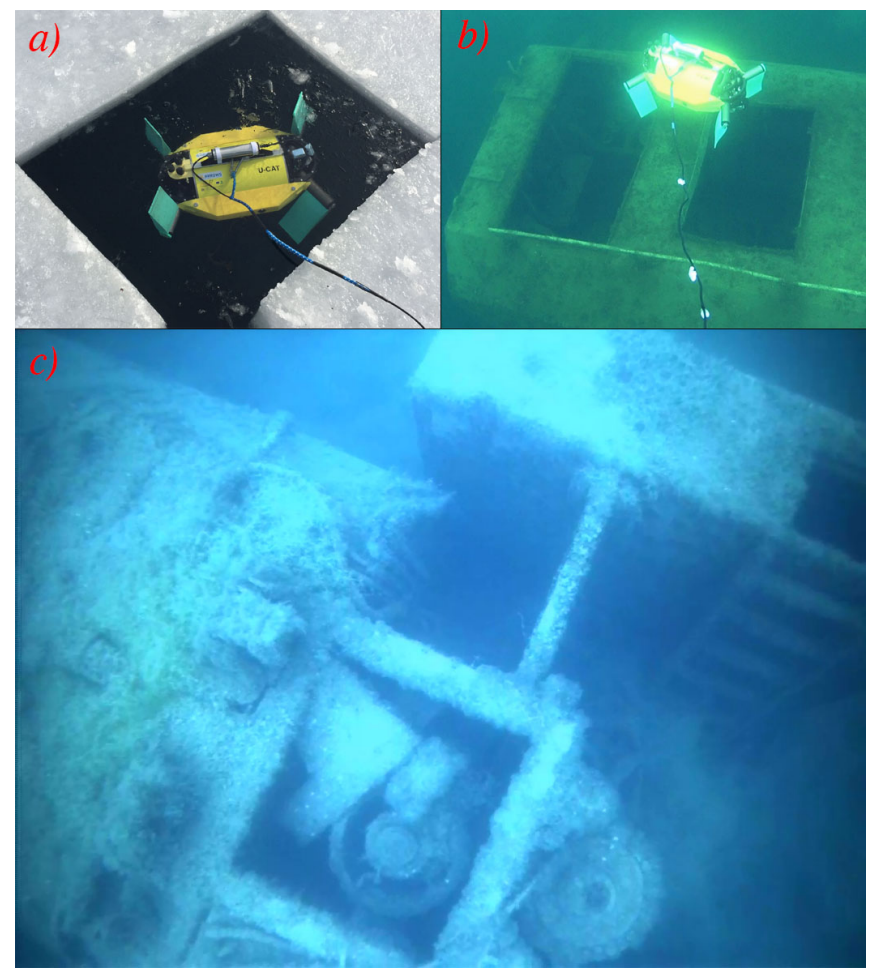

Fig. 15. Validating the control system in the real-life ROV inspection mission. a) U-CAT being launched through a hole in the ice; b) U-CAT hovering above the mining machine; c) snapshot of the U-CAT's on-board camera image.

of depth and yaw using incremental steps of $10 \mathrm{~cm}$ and $0.1 \mathrm{rad}$, while in the pool experiments smooth hyperbolic tangent trajectories were used. 

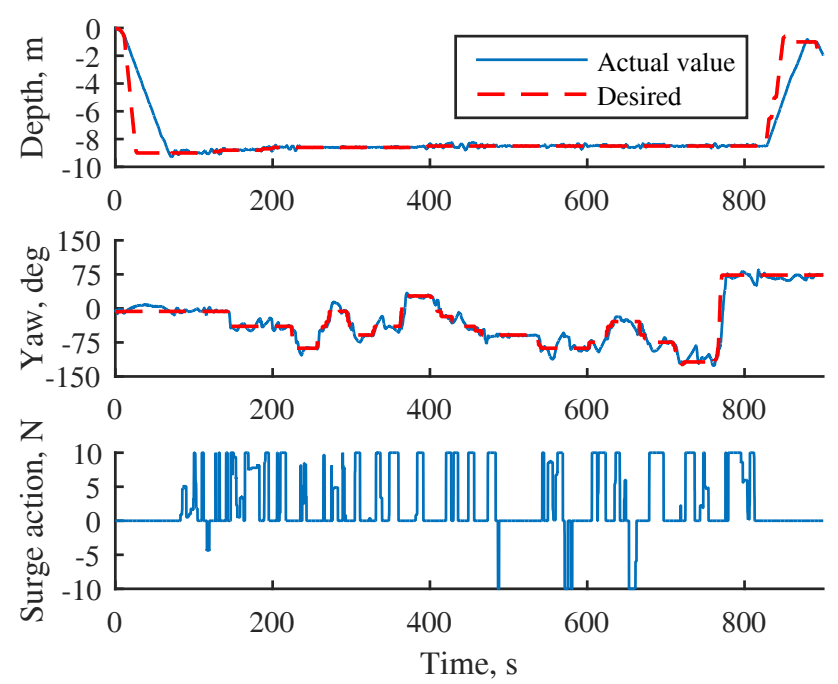

Fig. 16. Position tracking and surge action data during one of the dives of the inspection mission done at the Citadel.

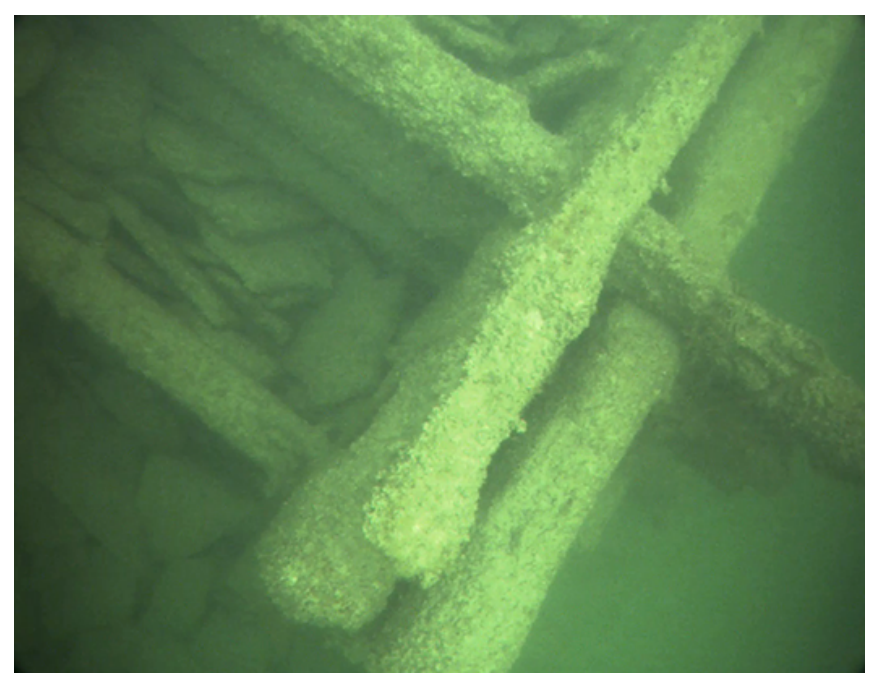

Fig. 17. View of the remains of the citadel fortifications in Tallinn Bay taken by the U-CAT's on-board camera

\section{B. Underwater Archaeology Mission on Citadel}

The second test site, the remains of the coastal defense battery known as The Citadel $\left(59^{\circ} 27^{\prime} 5.9 \mathrm{~N}\right.$ $24^{\circ} 47^{\prime} 27.8 \mathrm{E}$ ), located near the Tallinn shoreline and was chosen for its relevance to the application domain of U-CAT, underwater archaeology. The remains of the fortification are in 8-11 m depth about $900 \mathrm{~m}$ from shore. The archaeological interest was in inspecting the separate standing stone filled cellboxed built of wooden logs, in particular to document the possible damage by waves, ice and divers to the archaeological site. 
As opposed to the sheltered water site in Rummu quarry, Citadel is more exposed to currents and waves and visibility is poor due to turbidity of water and algae growth in summer. Here, the high maneuverability of U-CAT is particularly important in order to visually follow the complicated contours of the cell boxes at close distance while compensating for wave and current disturbances by the operator. The mission lasted 5 days, resulting in 11 hours of onboard camera footage. The robot was operated from a RIB (rigid-hulled inflatable boat). Due to the turbidity from the algae blooms and highly volatile silt rising from the bottom with currents, waves and motion of the robot and its tether, the visibility was only $1 \mathrm{~m}$ or less making close inspection of the logs of the cell boxes and its dove-tailed corners particularly challenging. The currents were estimated to reach about $0.5 \mathrm{~m} / \mathrm{s}$ (just about the max forward speed of U-CAT in hover mode) and the wave height was visually estimated about $0.5 \mathrm{~m}$.

Fig. 16 shows an example of a typical tracking data during one of many inspection dives. From the graph it can be seen that in the beginning and the end of the dive the operator modified the desired depth faster than the robot was able to move. Therefore, we calculate the tracking error only in the time range from the end of the initial dive to the beginning of the final resurface. The RMS error value for depth tracking is $6.16 \mathrm{~cm}$ and the RMS error of yaw tracking is $14.54^{\circ}$ ). Even though the depth tracking is slightly more precise and the yaw tracking less precise than in the experiments in sheltered quarry, the error values are still in similar range. This shows that the developed control system can operate with satisfying precision in more difficult conditions in the presence of environmental disturbances such as waves and currents.

\section{CONCLUSION}

This paper presented a new control architecture and its implementation for a biomimetic four-fin underwater robot U-CAT in hovering mode. Finned propulsion has several advantages compared to a traditional propeller driven underwater vehicle, in the case of the U-CAT high maneuverability on a cost of lower complexity, but it also proposes new challenges for control, mainly caused by strong motion coupling of the DOFs and delayed response of the fin actuators. We have built a modular control architecture and realised 2DOF and 3DOF control presenting a method of DOF priority selection. The tests show that this approach decreases the tracking errors of the U-CAT. We also subjectively experienced during the trials that it makes the control more intuitive for a human operator. Also the modular control architecture has been beneficial in the development phase allowing us to quickly change the control approaches (for example to 
switch between different 2DOF controllers). We therefore conclude that the architecture makes the robot easier to operate both in development phase as well as during real missions. The tests where carried out in controlled underwater environment, in sheltered water and in open water environment with waves, currents and very low visibility at a difficult inspection task. The tracking error increases when moving to more complicated environments, which is expected with any control approach. However, the control of the robot was sufficiently easy and precise even during a long real mission in a highly unstable environment. We therefore conclude that the proposed control architecture is suitable for U-CAT. Our future goal is to extend the control to more DOFs as well as to extend the motion library of U-CAT to include cruising mode. Again, we expect the modular control architecture to be beneficial in development and testing phase as well as on real underwater missions.

\section{ACKNOWLEDGMENT}

This research has received funding from the European Unions Seventh Framework Programme for Research technological development and demonstration, under grant agreement no. 308724 (The ARROWS Project), from Estonian- French joint collaboration project PHC-PARROT and from IUT339 grant of Estonian Ministry of Education and Research. The authors would like to thank Keijo Kuusmik, Jaan Rebane, Riho Markna who have helped to develop the UCAT vehicle. We would also like to thank TUT Small Craft Competence Centre for letting us use the tow tank.

\section{REFERENCES}

[1] B. Anderson and J. Crowell, "Workhorse auv-a cost-sensible new autonomous underwater vehicle for surveys/soundings, search \& rescue, and research," in OCEANS, 2005. Proceedings of MTS/IEEE. Washington, USA: IEEE, Sep. 2005, pp. $1-6$.

[2] (2016) Bluefin robotics website. [Online]. Available: http://www.bluefinrobotics.com/

[3] C. von Alt, "Remus 100 transportable mine countermeasure package," in OCEANS 2003. Proceedings, vol. 4. San Diego, USA: IEEE, 2003, pp. 1925-1930.

[4] A. Shukla and H. Karki, "Application of robotics in offshore oil and gas industry a review part \{II\}," Robotics and Autonomous Systems, vol. 75, Part B, pp. 508 - 524, 2016. [Online]. Available: http://www.sciencedirect.com/science/article/pii/S0921889015002018

[5] V. Yordanova and H. Griffiths, "Rendezvous point technique for multivehicle mine countermeasure operations in communication-constrained environments," Marine Technology Society Journal, vol. 50, no. 2, pp. 5-16, 2016.

[6] B. Bingham, B. Foley, H. Singh, R. Camilli, K. Delaporta, R. Eustice, A. Mallios, D. Mindell, C. Roman, and D. Sakellariou, "Robotic tools for deep water archaeology: Surveying an ancient shipwreck with an autonomous underwater vehicle," Journal of Field Robotics, vol. 27, no. 6, pp. 702-717, 2010. 
[7] Y.-S. Ryuh, G.-H. Yang, J. Liu, and H. Hu, "A school of robotic fish for mariculture monitoring in the sea coast," Journal of Bionic Engineering, vol. 12, no. 1, pp. 37-46, 2015.

[8] A. Kohl, K. Pettersen, E. Kelasidi, and J. Gravdahl, "Planar path following of underwater snake robots in the presence of ocean currents," Robotics and Automation Letters, IEEE, vol. PP, no. 99, pp. 1-1, 2016.

[9] R. J. Lock, R. Vaidyanathan, S. C. Burgess, and J. Loveless, "Development of a biologically inspired multi-modal wing model for aerial-aquatic robotic vehicles through empirical and numerical modelling of the common guillemot, uria aalge," Bioinspiration \& biomimetics, vol. 5, no. 4, p. 046001, 2010.

[10] M. Kemp, B. Hobson, and J. H. Long, "Madeleine: an agile auv propelled by flexible fins," in Proceedings of the 14th International Symposium on Unmanned Untethered Submersible Technology, vol. 6, 2005.

[11] S. Licht and N. Durham, "Biomimetic robots for environmental monitoring in the surf zone \& in very shallow water," in IEEE/RSJ International Conference on Intelligent Robots and Systems, Vilamoura-Algarve, Portugal, Oct. 2012.

[12] G. Yao, J. Liang, T. Wang, X. Yang, Q. Shen, Y. Zhang, H. Wu, and W. Tian, "Development of a turtle-like underwater vehicle using central pattern generator," in Robotics and Biomimetics (ROBIO), 2013 IEEE International Conference on. Shenzhen, China: IEEE, Dec. 2013, pp. 44-49.

[13] G. Dudek, P. Giguere, J. Zacher, E. Milios, H. Liu, P. Zhang, M. Buehler, C. Georgiades, C. Prahacs, S. Saunderson et al., “Aqua: An amphibious autonomous robot," Computer, no. 1, pp. 46-53, 2007.

[14] A. Konno, T. Furuya, A. Mizuno, K. Hishinuma, K. Hirata, and M. Kawada, "Development of turtle-like submergence vehicle," in Proceedings of the 7th International Symposium on Marine Engineering, 2005.

[15] K. Low, C. Zhou, T. Ong, and J. Yu, "Modular design and initial gait study of an amphibian robotic turtle," in Robotics and Biomimetics, 2007. ROBIO 2007. IEEE International Conference on. Sanya, China: IEEE, Dec. 2007, pp. 535-540.

[16] S. C. Licht, "Biomimetic oscillating foil propulsion to enhance underwater vehicle agility and maneuverability," Ph.D. dissertation, Massachusetts Institute of Technology, Cambridge, Massachusetts, June 2008.

[17] J. D. Geder, R. Ramamurti, D. Edwards, T. Young, and M. Pruessner, "Development of a robotic fin for hydrodynamic propulsion and aerodynamic control," in Oceans-St. John's, 2014 . IEEE, 2014, pp. 1-7.

[18] W. Zhao, Y. Hu, L. Wang, and Y. Jia, "Development of a flipper propelled turtle-like underwater robot and its cpg-based control algorithm," in Decision and Control, 2008. CDC 2008. 47th IEEE Conference on. Cancun, Mexico: IEEE, Dec. 2008, pp. 5226-5231.

[19] C. Siegenthaler, C. Pradalier, F. Gunther, G. Hitz, and R. Siegwart, "System integration and fin trajectory design for a robotic sea-turtle," in Intelligent Robots and Systems (IROS), 2013 IEEE/RSJ International Conference on. Tokyo, Japan: IEEE, Nov. 2013, pp. 3790-3795.

[20] J. D. Geder, R. Ramamurti, M. Pruessner, and J. Palmisano, "Maneuvering performance of a four-fin bio-inspired uuv," in Oceans-San Diego, 2013. IEEE, 2013.

[21] C. Wang, G. Xie, X. Yin, L. Li, and L. Wang, "Cpg-based locomotion control of a quadruped amphibious robot," in Advanced Intelligent Mechatronics (AIM), 2012 IEEE/ASME International Conference on. IEEE, 2012.

[22] A. Chemori, K. Kuusmik, T. Salumäe, and M. Kruusmaa, "Depth control of the biomimetic u-cat turtle-like auv with experiments in real operating conditions," in Robotics and Automation (ICRA), 2016 IEEE International Conference on. Stockholm, Sweden: IEEE, May 2016, pp. 1-6.

[23] T. Salumäe, A. Chemori, and M. Kruusmaa, "Motion control architecture of a 4-fin u-cat auv using dof prioritization," in Intelligent Robots and Systems (IROS), 2016 IEEE/RSJ International Conference on. Daejeon, Korea: IEEE, Oct. 2016.

[24] J. Wyneken, "Sea turtle locomotion: mechanisms, behavior, and energetics," in The biology of sea turtles, P. L. Lutz and J. A. Musick, Eds. Boca Raton, FL: CRC Press, 1997, ch. 7, pp. 165-198. 
[25] S.-H. Song, M.-S. Kim, H. Rodrigue, J.-Y. Lee, J.-E. Shim, M.-C. Kim, W.-S. Chu, and S.-H. Ahn, "Turtle mimetic soft robot with two swimming gaits," Bioinspiration I\& Biomimetics, vol. 11, no. 3, p. 036010, 2016. [Online]. Available: http://stacks.iop.org/1748-3190/11/i=3/a=036010

[26] J. H. Long Jr, J. Schumacher, N. Livingston, and M. Kemp, "Four flippers or two? tetrapodal swimming with an aquatic robot," Bioinspiration \& Biomimetics, vol. 1, no. 1, p. 20, 2006.

[27] N. Plamondon and M. Nahon, "A trajectory tracking controller for an underwater hexapod vehicle," Bioinspiration \& biomimetics, vol. 4, no. 3, p. 036005, 2009.

[28] N. Plamondon, "Modeling and control of a biomimetic underwater vehicle," Ph.D. dissertation, McGill University, Montreal,Quebec, January 2010.

[29] P. Giguere, Y. Girdhar, and G. Dudek, "Wide-speed autopilot system for a swimming hexapod robot," in Computer and Robot Vision (CRV), 2013 International Conference on. Regina, Canada: IEEE, May 2013, pp. 9-15.

[30] D. Meger, F. Shkurti, D. Cortes Poza, P. Giguere, and G. Dudek, "3d trajectory synthesis and control for a legged swimming robot," in Intelligent Robots and Systems (IROS 2014), 2014 IEEE/RSJ International Conference on. Chicago, USA: IEEE, Sep. 2014, pp. 2257-2264.

[31] B. Allotta, R. Costanzi, A. Ridolfi, C. Colombo, F. Bellavia, M. Fanfani, F. Pazzaglia, O. Salvetti, D. Moroni, M. A. Pascali et al., "The arrows project: adapting and developing robotics technologies for underwater archaeology," IFACPapersOnLine, vol. 48, no. 2, pp. 194-199, 2015.

[32] T. Salumäe, R. Raag, J. Rebane, A. Ernits, G. Toming, M. Ratas, and M. Kruusmaa, "Design principle of a biomimetic underwater robot u-cat," in Oceans-St. John's, 2014. IEEE, 2014, pp. 1-5.

[33] T. I. Fossen, Handbook of marine craft hydrodynamics and motion control. John Wiley \& Sons, 2011.

[34] H. E. Daou, T. Salumäe, L. D. Chambers, W. M. Megill, and M. Kruusmaa, "Modelling of a biologically inspired robotic fish driven by compliant parts," Bioinspiration \& Biomimetics, vol. 9, no. 1, p. 016010, 2014. [Online]. Available: http://stacks.iop.org/1748-3190/9/i=1/a=016010

[35] T. I. Fossen and $\varnothing$. N. Smogeli, "Nonlinear time-domain strip theory formulation for low-speed manoeuvering and stationkeeping," Modeling, identification and control, vol. 25, no. 4, p. 201, 2004.

[36] P. Chiacchio, S. Chiaverini, L. Sciavicco, and B. Siciliano, "Closed-loop inverse kinematics schemes for constrained redundant manipulators with task space augmentation and task priority strategy," The International Journal of Robotics Research, vol. 10, no. 4, pp. 410-425, 1991.

[37] W. Wang, PID Controller Design Approaches - Theory, Tuning and Application to Frontier Areas, M. Vagia, Ed. InTech, 2012.

[38] W. Shang and S. Cong, "Nonlinear computed torque control for a high-speed planar parallel manipulator," Mechatronics, vol. 19, no. 6, pp. 987-992, Sep. 2009.

[39] — Serial and Parallel Robot Manipulators - Kinematics, Dynamics, Control and Optimization, Dr. Serdar Kucuk (Ed.). InTech, 2010, ch. 12. 